VI.

\title{
Ueber die operativen und functionellen Erfolge der Operation der angeborenen Gaumenspalte.
}

\author{
Von \\ Dr O. Kappeler, \\ Chefarzt des stadt. Krankenhauses in Konstanz.
}

Der Ausspruch Küsters'), dass wir noch keine Statistik dessen, was bisher durch die operative Behandlung der angeborenen Gaumenspalte geleistet worden, besitzen, besteht heute noch zu Recht. Die bisherigen Mittheilungen über dieses ausserordentlich wichtige und dankbare Capitel der operativen Chirurgie beschränken sich zumeist auf Mittheilung einzelner Fälle, auf Neuerungen und Verbesserungen der Operationsmethoden, und ausser den Mittheilungen Ehrmann's, Küster's und Dobberkau's aus der v. Bergmann'schen Klinik liegen heute noch keine neueren und vollständigen Gesammterfahrungen einzelner Chirurgen oder Kliniker vor. ${ }^{2}$ ) Die neueste Publication J. Wolffs ${ }^{3}$ ), dessen grosse Verdienste um die Ausgestaltung und Vorvollkommnung des v. Langenbeck'schen Verfahrens der Gaumenspaltenoperation Niemand verkennen wird und der wohl über die grösste Zahl von Operationen verfügt, giebt zwar eine kurze tabellarische Zusammenstellung seiner operativen Resultate, aber die Mittheilungen über die funetionellen Erfolge beziehen sich nur auf wenige, allerdings durch vortreffliche Schlussresultate ausgezeichnete, Fälle. Auf diese Weise können wir aber kein vollständiges Bild über die Leistungsfähigkeit der Gaumenspaltenoperation gewinnen. Dies kann nur geschehen durch rückhaltlose und vollständige Mittheilung auch der functionellen Erfolge. Bd. 46 .

1) Küster, Operative Behandlung der Gaumenspalten. Langenbeck's Archiv.

2) Eine Arbeit von $\mathrm{Kassel}$ aus der Breslauer chirurgischen Klinik kam mir erst nach Fertigstellung des Manuseriptes zu Gesicht und konnte nicht mehr benützt werden.

3) Ueber frühzeitige Operation der angeborenen Gaumenspalten von J. Wolff, Sammlung klinischer Vorträge. 
Es soll daher, um die kleine Serie vollständiger Gesammterfahrungen zu ergänzen und zu vervollständigen, hier über 82 Fälle von mir operirter Gaumenspalten berichtet werden, wobei ich in der glücklichen Lage bin, über das operative Resultat und das spätere Schicksal aller Operirten Auskunft geben und mit Ausnahme von 3 Fällen den functionellen Erfolg theils durch eigene Nachuntersuchung, theils durch zuverlässige Nachrichten seitens der Operirten und ihrer Aerzte, endgiltig feststellen zu können.

Meine Beobachtungen und Operationen beziehen sich auf Kinder verschiedensten Alters und auf Erwachsene bis zu 40 Jahren. Sie werden deshalb, um eine leichte Uebersicht zu gewinnen, in versehiedene Gruppen abgetheilt, und zwar umfasst die 1. Gruppe die Kinder unter 2 Jahren, die 2. Gruppe die Kinder von 2-6 Jahren, die 3. die Kinder von 7-14 Jahren und die 4. alle Operirten von 15-40 Jahren.

In der beffolgenden Tabelle sind dis operativen Resultate der Uranostaphylorrhaphie bei den Kindern unter 2 Jahren (von 17 Tagen bis zu 23 Monaten) zusammengestellt:

\begin{tabular}{|c|c|c|c|c|c|}
\hline \multirow[b]{2}{*}{ Art der Spalte } & \multicolumn{2}{|c|}{$\begin{array}{c}\text { Vollkommener Verschluss } \\
\text { der Spalte }\end{array}$} & \multirow{2}{*}{ 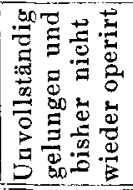 } & \multirow{2}{*}{ 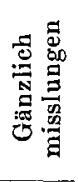 } & \multirow{2}{*}{$\begin{array}{l}\frac{a}{0} \\
\frac{0}{0} \\
\frac{0}{0} \\
00 \\
0 \\
0\end{array}$} \\
\hline & $\begin{array}{l}\text { durch eine } \\
\text { einzige } \\
\text { Operation }\end{array}$ & $\begin{array}{l}\text { mit Hilfe } \\
\text { von Nach- } \\
\text { operationen }\end{array}$ & & & \\
\hline $\begin{array}{l}\text { Spaltung des weichen Ganmens } \\
\text { uod der horizontalen Platte des }\end{array}$ & $?$ & & . & & \\
\hline $\begin{array}{l}\text { Bis zur Mitte des Process. palatin. } \\
\text { des Oberkiefers }\end{array}$ & 1 & - & - & - & - \\
\hline $\begin{array}{l}\text { Spaltung des ganzen harten Gau- } \\
\text { mens mit Ausnahme des Al- } \\
\text { veolarfortsatzes }\end{array}$ & - & - & - & 1 & - \\
\hline Durchgehende rechtsseitige Spalte & - & 1 & 1 & - & - \\
\hline Durchgehende linksseitige Spalte & 2 & 1 & 2 & - & - \\
\hline Bilateralspalte. . . . . . & - & - & - & 1 & - \\
\hline
\end{tabular}

Zur näheren Erläuterung der Tabelle diene Folgendes:

Von den 5 Kindern, die durch eine einzige Operation geheilt wurden, leben heute noch 3. Von den zweien, die seither gestorben sind, erlag das eine 3 Wochen nach der Operation zu Hause einem Erysipel, das von, am Nacken und Kopf aufgetretenen, Furunkeln ausging, das andere 6 Wochen nach der Operation, laut Bericht des behandelnden Arztes, einer Lungenentzündung.

Von den mit Hilfe von Nachoperationen von ihrer Gaumenspalte befreiten Kindern starb ebenfalls eines, und zwar 1 Jahr nach der Operation, an Meningitis, ohne Zweifel tabereulöser Natur. 
Von den Kindern, bei denen die Operation nur unvolltsändig gelang und die sich seither zu einer Nachoperation nicht verstehen konnten, starb eines (es konnte nur die Vereinigung des harten Gaumens erreicht werden) 3 Jahre nach der Operation an Diphtherie, bezüglich der 2 noch lebenden wurde bei einem der Kinder wegen grosser Gebrechlichkeit und Schwächlichkeit nur die Uranoplastik ohne Staphylorrhaphie gemacht und beim zweiten gelang von der Uranostaphylorrhaphie nur die Vereinigung des harten Gaumens.

Auch bei den Kindern mit gänzlich misslungener Operation ist ein Todesfall, und zwar, 1/4 Jahr nach der Operation, an katarrhalischer Pneumonie, laut ärztlichem Bericht, zu verzeichnen, so dass also heute von den 12 Kindern nur noch 7 leben.

Wir kommen zu functionellen Resultaten bei diesen noch lebenden 7 Kindern. Dieser functionelle Erfolg ist selbstverständlich sowohl bei dem Kinde, dessen Operation cinen vollständigen Misserfolg hatte, als auch bei den 2 Kindern, wo nur die Vereinigung des harten Gaumens zu Stande kam, gleich Null, das heisst, die Sprache bleibt undeutlich und stark näselnd.

Von den tibrigbleibenden 4 Kindern betrifft der erste Fall eine durchgehende linksseitige Spalte, die erst im 21. Lebensjahre zum vollständigen Schluss gebracht wurde. Bei dem Kranken, der niemals Sprechunterricht hatte, der aber eine Zeit lang einen Rachenobturator trug, wurde die Sprache deutlich, blieb aber näselnd. Bei 2 Kindern (das eine hatte eine Spalte des weichen Gaumens mit Spaltung der horizontalen Platte des Gaumenbeins, beim andern reichte die Spalte bis zur Mitte des Process. palatin. des Oberkiefers), die ebenfalls keinen systematischen Sprechunterricht hatten, bleibt die Sprache näselnd und undeutlich, doch stellt bei einem dieser Kinder der Sprachlehrer nach vorgenommener Prüfung der Sprache, durch sachgemässen Sprechunterricht allein ohne Zuhilfenahme eines Obturators, eine normale Sprache in sichere Aussicht.

Bei dem letzten Kinde dieser Serie, das im 7. Monat an einer Spalte des weichen Gaumens und der horizontalen Platte des Gaumenbeins erfolgreich operirt worden war, ist die Sprache ohne Sprechunterricht und ohne Obturator normal geworden. Ich lasse die Krankengeschichte dieses Kindes hier folgen:

0., Maria, 7 Monate altes Kind noch lebender gesunder Eltern, in deren Familie weder väterlicher- noch mütterlicherseits Hasenscharten, Gaumenspalten oder andere Bildungsfehler vorkamen, kam mit einer Spalte des weichen Gaumens und der horizontalen Platte des Gaumenbeins, deren grösste Breite $9 \mathrm{~mm}$ beträgt, zur Welt. Der Aufnahme in's Spital am 
22. August 1889 folgte am 24. August die Operation der Gaumenspalte. Zur Abkürzung der Narkose wird zunächst die Anfrischung der Spaltenränder ohne Chloroform vorgenommen, und zwar am hängenden Kopf mit Whitehead'schem Speculum. Dann in Chloroformnarkose Loslösung der seitlichen Lappen und Naht. Es werden 7 Nälıte, 2 an der Hinterseite der Uvula, angelext. In die klaffenden Seitenselnitte kommen kleine Jodoformmèchen, Vom 25.-29. August starke Schleim- und Speichelsecretion und erhöhte Abendtemperaturen $\left(38,2,38,3,38,4,35,8^{\circ}\right.$ C.). Entfernung der Nälte am 7. Tage in Narkose, wobei der vollständige Schluss der Spalte constatirt wird.

In seinem 12. Lebensjahre sah ich das Mädchen wieder, Mai 1901. Es wuchs auf wie andere Kinder und hatte niemals besonderen Sprachunterricht, besuchte die öffentliche Schule und lernte gut. Von den Mitschülerinnen wurde es niemals wegen abnormen Sprechens ausgelacht, ja weder dem Jehrer noch den Mitschiilerinnen soll aufgefallen sein, dass es anders spreche, als andere Kinder. Der weiche Gaumen hat jetzt eine Höhe von $24 \mathrm{~mm}$ und ist wohlgebildet mitsammt der etwas kurzen Uvula, Pharynxwulst nicht auffallend stark entwickelt. Beim Intoniren von A helot sich das granze Velum rasch und kräftig nach oben und hinten und die oberste und mittlere Partie desselben steht dabei fast horizontal. Beim zusammenhängenden Sprechen, beim Vollesen und Singen, hat die Stimme viel Klang: und ist normal, $d$ h. man merkt ilı weiter nichts an. Eine sorgfältige Sprachprüfung ergiebt dann allerdings, dass nur ab und zu bei der Intonation des $I$ in zusammengesetzten Worten ein kaum bemerkbarer, nasaler Beiklang zu lıören ist, wälırend alle übrigen Vocale absolut rein lauten, dass die 'Tenues alle rein sind und von den Mediae nur das b einen ganz leichten Nasendurchschlag zeigt und dass ein deutliches Zungen-R vorhanden ist. Es ist ohne Weiteres klar, dass auch diese leichten, das Sprechen nicht beeinträchtigenden, Sprachstörungen durch einige Sprechübungen loicht beseitigt werden könnten.

Die Resultate der Uranostaphylorrhaphie bei Kindern von 2 bis 6 Jahren sind in folgender Tabelle zusammengestellt:

\begin{tabular}{|c|c|c|c|c|c|}
\hline \multirow[b]{2}{*}{ Art der Spalte } & \multicolumn{2}{|c|}{$\begin{array}{c}\text { Vollkommener Versehluss } \\
\text { der Spalte }\end{array}$} & \multirow{2}{*}{ 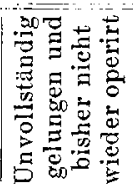 } & \multirow{2}{*}{ 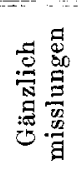 } & \multirow{2}{*}{ 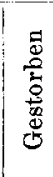 } \\
\hline & $\begin{array}{l}\text { durch eine } \\
\text { einzige } \\
\text { Operation }\end{array}$ & $\begin{array}{l}\text { mit Hilfe } \\
\text { von Nach- } \\
\text { operationen }\end{array}$ & & & \\
\hline Spaltung des weichen Gaumens & 1 & - & - & 1 & - \\
\hline $\begin{array}{l}\text { Spaltung des weichen Gaumens } \\
\text { und der horizontalen Platte des } \\
\text { Gaumenbeins } . . \\
\text {. . . . . . }\end{array}$ & 2 & - & - & - & - \\
\hline $\begin{array}{l}\text { Spaltuug des Gaumens bis zur } \\
\text { Mitte des Process. palatin. des } \\
\text { Oberkiefers. . . . . . . }\end{array}$ & - & 2 & - & -- & - \\
\hline $\begin{array}{l}\text { Spaltung des ganzen Gaumens } \\
\text { mit Ausuahme des Alveolar- } \\
\text { fortsatzes. . . . . . }\end{array}$ & 1 & 1 & - & - & 一 \\
\hline Durchgehende linksseitige Spalte & - & 2 & -- & 1 & \\
\hline
\end{tabular}


Diese 11 Kinder, von denen 3 schon in den Jahren 1881, 1887 und 1889 operirt wurden, leben heute alle noch.

Was nun den functionellen Erfolg betrifft, so ist bei keinem der selben die Sprache ohne weiteres Dazuthun normal geworden. Bei den 2 misslungenen Operationen ist der Erfolg bezüglich Verbesserung der Sprache gleich Null.

Die Uebrigen sind zu scheiden in solche, die systematischen Sprechunterricht nahmen, in solche, die keinen solchen nahmen und endlich in solche, die wegen zu jugendlichen Alters noch keinen Sprechunterricht nehmen konnten.

Die letzteren - es sind deren 4 - haben ein Alter von 3, 4, $41 / 2$ und 6 Jahren und sollen nach meinem und der Eltern Wunsch sobald als möglich dem Sprachlehrer übergeben werden.

In diesen 4 Fällen kann somit von einem abschliessenden Resultat noch keine Rede sein. 2 der Operirten, K. Peter, und N. Bertha hatten niemals Sprechunterricht. Der erstere, jetzt 20 jähriger Schmied, nebenbei ein geistig beschränkter Mensch, soll deutlich, aber noch nasal, sprechen, die zweite, ein jetzt 12jähriges Mädchen, zeigt keine wesentliche Besserung der Sprache seit der Operation.

3 hatten Sprechunterricht: der Erste, J., Hans, der mit $5^{3 / 4}$ Jahren wegen durchgehender linksseitiger Gaumenspalte operirt worden war, hatte unmittelbar nach der Operation ohne Obturator 6 Wochen Sprechunterricht mit geringem Erfolg, dann im 8. Lebensjahr nochmals $1 \mathrm{Jahr}$ lang, wöchentlich 1 Stunde. Nach schriftlicher Mittheilung des Vaters spricht der jetzt 11 jährige Knabe verhältnissmässig recht gut, wenn er langsam spricht und sich Mühe giebt. Die Stimme ist klangvoll ohne jeden näselnden Beiklang.

Die Vocale sind ohne Ausnahme von tadellosem Klang, ebenso die meisten Consonanten, mit Ausnahme des G und S. Der Knabe soll später nocbmals Unterricht bekommen, so dass eine normale Sprache in Aussicht steht.

Die Zweite, L., Christine, die mit 31/2 Jahren wegen Spaltung des ganzen harten Gaumens mit Ausnahme des Alveolarfortsaizes operirt worden war und die eine nasale Sprache behalten hatte, nimmt auf mein Drängen im Alter von 15 Jahren einen nur 14tägigen Sprechunterricht mit dem glänzenden Erfolg, dass die Sprache in dieser verhältnissmässig kurzen Zeit völlig normal wird.

Die drittoperirte Kranke mit Spaltung bis zur Mitte des harten Gaumens, die mit 5 Jahren operirt worden war, nimmt mit immer noch nasaler und schwerverständlicher Sprache einen 5 wöchentlichen Sprechunterricht ohne Obturator. Herr Schitten müller schreibt über 
das Resultat: Schon nach 6 Uebungsstunden waren alle einfachen Consonanten, ja das Zungenspitzen- $R$ da, die zusammengesetzten Laute Z, K machten weiter gar keine Schwierigkeiten, wie auch die Vocale I und $\mathrm{U}^{\top}$ von Tag zu Tag an Reinheit gewinnen. Nach 80 Uebungsstunden ( 5 Wochen) spricht das Mädchen absolut rein, natürlich und auch ziemlich fliessend, und zwar in ihrem ziemlich schwierigen Dialect ebensogut, wie im Schriftdeutschen. Auch singt sie sehr hübsch.

In folgender Tabelle sind die operativen Resultate der Uranostaphylorrhaphie bei Kindern von 6-14 Jahren übersichtlich zusammengestellt:

\begin{tabular}{|c|c|c|c|c|c|}
\hline \multirow[b]{2}{*}{ drt der Spalte } & \multicolumn{2}{|c|}{$\begin{array}{l}\text { Vollkommener Verschluss } \\
\text { der Spalte }\end{array}$} & \multirow{2}{*}{ 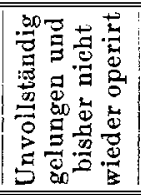 } & \multirow{2}{*}{ 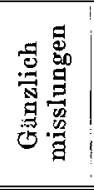 } & \multirow{2}{*}{ 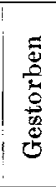 } \\
\hline & $\begin{array}{l}\text { durch eine } \\
\text { einzige } \\
\text { Operation }\end{array}$ & $\begin{array}{l}\text { mit Hilfe } \\
\text { von Nach. } \\
\text { operationen }\end{array}$ & & & \\
\hline Spaltung des weichen Gaumens & 7 & 3 & 1 & - & - \\
\hline $\begin{array}{l}\text { Spaltung des weichen Gaumens } \\
\text { und der horizontaten Platte des }\end{array}$ & & & & & \\
\hline Gaumenbeins . . . . . . & ) & - & 1 & - & - \\
\hline $\begin{array}{l}\text { Spaltung des Gaumens bis zur } \\
\text { Mitte des Process. palatin, des }\end{array}$ & & & & & \\
\hline Oberkiefers . . . . . & 2 & 2 & - & - & 一 \\
\hline Durchgehende rechtsseitige Spalte & 1 & 1 & - & - & 一 \\
\hline Durchgehende linksseitige Spalte & 1 & 3 & $\ldots$ & - & - \\
\hline
\end{tabular}

Von diesen 27 Kindern ist seither nur eins gestorben, und zwar 1 Jahr nach der Operation an Epityphlitis. Ein zweites ist nach Amerika ausgewandert und es konnten in diesem Fall über das functionelle Resultat keine Erhebungen gemacht werden. Dazu kommen noch 2 weitere Fälle mit nicht ganz vereinigten Spalten, die aus zufälligen, mit dem Leiden in keinem Zusammenhang stehenden, Gründen noch nicht durch eine zweite Operation geschlossen werden konnten.

Dagegen können wir Auskunft geben über das functionelle Resultat bei den noch übrig bleibenden 23 Fällen, und da möge vor Allem die erfreuliche Thatsache mitgetheilt werden, dass bei zweien dieser 23, beide mit angeborenen Spalten des weichen Gaumens, ohne besonderen Sprechunterricht und ohne Prothese irgend welcher Art die Sprache gewissermaassen von selbst eine ganz normale geworden ist. Ich lasse die Krankengeschichten dieser 2 Fälle hier folgen.

1. W., Hermann, 11 Jahre alt, wurde am 4. December 1890 in's Spital aufgenommen. In der Familie des Knaben sind keine Hasenscharten, Deutsche Zeitschrift f. Chirurgie. LXVII, Bd. 
Gaumenspalten oder andere Missbildungen zur Beobachtung gekommen. Die Gaumenspalte des Kindes wurde erst entdeckt, als es zu sprechen anfing, im Alter von 1.3/4 Jahren. Das Kind sprach stark näselnd, anfänglich fast unverständlich, in ilen letzten Jahren vor dem Spitaleintritt soll die Sprache, ohne das näselnde Timbre zu verlieren, etwas deutlicher geworden sein. Auch Schluekbeschwerten waren rorhanden. Die Bissen blieben im Halse stecken und das Kind musste stark würgen, um sie lielunter zu bringen.

Gut entwickelter Knabe ron gesundem Aussehen. Fs besteht eine durch den ganzen weichen Gaumen median verlaufende Spalte, die mit al,gerundetem Winkel am hinteren Rand des harten Gaumens endet. Breite der Spalte zwisehen den Zäpfehenlıälften $17 \mathrm{~mm}$, in der Mitte 14, vorn 7 .

Die Sprache ist ausgesprochen nasal. Zusammenlıängendes Sprechen und Vorlesen ist kaum verständlich. Sämmtliche Vocale nasal, b gleich me, $\mathrm{d}, \mathrm{tz}$ mit starkem Nasendurchschlag, g und $\mathrm{k}$ sind nicht rorhanden.

Am 8. December 1890 Uranostaphylorrhaphie am längenden Kopf in Chloroformnarkose mit Bill roth'seher Abmeisselmng des Amul. pterygoirl. 8 Nälıte, 2 an der Hinterseite der Uvula. Am 16. nach fieberlosem Verlauf Entfernung der Nähte. Vollständige Vereinigung der Spalte mit Ausnahme der Zäpfelienspitze, so dass eine Uvula bifila zurüekbleiltt.

Der Knabe besuchte nach der Operation noch 3 Jahre die Schule und 2 Jahre die Fortbildungsschule. Die Sprache muss damals noch mangelhaft gewesen sein, demn der Lehrer soll ilm heim Vorlesen oft nicht verstanden haben. Später beschäftigte er sich mit Landwirthschaft. Am 12. October 1900 wurde er zum Militär eingezogen und von der Aushebungscommission untersucht. Er wurde ganz gesund befunden und nichts Abnormes an seiner Sprache entdeckt, weshalb er berlingungslos als Soldat eingereiht wurde. Seither diente er als Soldat und während des Dienstes (seit $11 / 2$ Jahren) wurde weder von Offizieren noch von Soldaten die Beobaclitung gemacht, dass er anders spreche, als seine Kameraden. Zufälliz" entdeekte der mir befreundete Oherstabsarzt Dr. Ja eckel die Narbe am Gaumen und machte mich auf den Mann aufmerksam. Ich liess iln kommen und fand einen gut entwickelten, sich ausgiebig und kräftig bewegenden, weichen Ganmen mit gespaltener Zäpfchenspitze. Die Sprache ist normal, d. h. man merkt beim Sprechen und Vorlesen nichts Abnormes unil nur bei grosser Aufmerksamkeit entdeckt man an dem Vocal $\mathbf{I}$ in zusammengesetzten Worten einen Anflug nasalen Timbres. Alle übrigen Vocale sind rein, ebenso alle Consonanten mit Ausnahme des K, das fehilt, das ilm aber, wie der Sprachlehrer meint, in einigen Unterrichtsstunden beigebracht werden könnte.

Der Mann wurde der ärztlichen Gesellschaft Münsterlingia vorgestellt.

2. Die 14 jälırige 13., Itla, wurde am 13 . August 1890 in's Spital aufgenommen. Kräftiges, blühend aussehendes, Mädchen, das mit einer angeborenen Spalte des weichen Gaumens helaftet ist und eine selrr undeutliche, mühsam sich anhörende und stark nasale Sprache hat. Beim Vorlesen längerer Perioden sind meist nur vereinzelte Worte verständlich.

Am 14. August Uranostaphylorthaphie. Anfrischung. der Spaltenränder unter Cocainbepinselung, die übrige Operation in Chloroformnarkose am hängenden Kopf. 9 Nähte, 2 am Zäpf́chen, die am 6., 7. und 8. Tage entfernt werden. Die vollständige Vereinigung gelingt auf's Schönste. Am 23. Mai 1901 stellt sich das Mädchen wieder vor. Es findet sich ein wohl- 
yebildetes Velum mit sehön geschwungenen Arcus palatopharyngi und palatoglossi und mit normalem Zäpfchen. Bei der Intonation von A wird das Velum kräftig in die Iöhe gezogen. Mässig entwickelter Pharynxwulst.

Obwohl das Mädehen nie Sprachunterricht hatte und nie eine Prothese beniitzte, spricht und liest sie mit klangvoller Stimme gelïufig olne eine Spur von Näseln. Sämntliche Vocale sind rein, desgleichen alle Consonanter, auch $G$ und $K$, sowie die beiden R. Singt mit reiner Stimme.

Patientin wurle dem ärztlichen Kreisverein Konstanz vorgestellt.

6 Kranke bedienten sich weder einer Prothese noch nahmen sie besonderen Sprechunterricht. Bei der Mehrzahl derselben wurde die Sprache einige Zeit nach der Operation etwas deutlicher, behielt aber ausnahmslos den ausgesprochenen nasalen Charakter.

11 der Kranken erhielten mit Zuhilfenahme des Brugger'schen Obturators systematischen Sprechunterricht durch die Herren Sch ittenmüller und Wang, der 1 mal nur 3 Wochen, 4 mal 4 Wochen, 1 mal 5 Wochen, 1 mal 6 Wochen, 2 mal 7 Wochen, 1 mal 10 Wochen und bei einem schwerhörigen, schwächlichen Knaben 6 Monate dauerte. Die Sprache wurde in allen Fällen durchaus normal.

9 dieser Kranken haben später ohne Nachtheil für die Sprache den Obturator abgelegt.

4 der Operirten endlich bekamen durch Sprechunterricht allein, der 4, 6, 8 und 12 Wochen dauerte, eine normale, reine Sprache.

Ueber die operativen Resultate der Uranostaphylorrhaphie bei Individuen von 15-40 Jahren giebt folgende Tabelle Aufschluss:

\begin{tabular}{|c|c|c|c|c|c|}
\hline \multirow[b]{2}{*}{ Art der Spalte } & \multicolumn{2}{|c|}{$\begin{array}{l}\text { Vollkommener Verschluss } \\
\text { der Spalte }\end{array}$} & \multirow{2}{*}{ 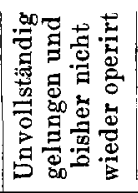 } & \multirow{2}{*}{ 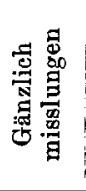 } & \multirow{2}{*}{ 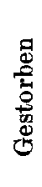 } \\
\hline & $\begin{array}{l}\text { durch eine } \\
\text { einzige } \\
\text { Operation }\end{array}$ & $\begin{array}{c}\text { mit Hilfe } \\
\text { von Nach- } \\
\text { operationen }\end{array}$ & & & \\
\hline $\begin{array}{l}\text { Längsovaler Defect des harten } \\
\text { Gaumens. . } . . \\
.\end{array}$ & 1 & - & - & - & - \\
\hline Spaltung des weichen Gaumens & 5 & - & - & - & - \\
\hline $\begin{array}{l}\text { Spaltung des weiehen Gaumens } \\
\text { und der horizontalen Platte des } \\
\text { Gaumenbeins . . . . . . }\end{array}$ & 7 & 3 & 1 & - & - \\
\hline $\begin{array}{l}\text { Spaltung des Gaumens bis zur } \\
\text { Mitte des Process. palatin. des } \\
\text { Oberkiefers........ . }\end{array}$ & 1 & 1 & - & - & - \\
\hline $\begin{array}{l}\text { Spaltung des ganzen Gaumens } \\
\text { mit Ausnahme des Alveolar- } \\
\text { fortsatzes . . . . . . . . }\end{array}$ & 1 & 2 & 1 & - & - \\
\hline Bilateralspalte des Ganmens . . & - & 1 & - & - & - \\
\hline Durchgehende rechtsseitige Spalte & 2 & 2 & $\rightarrow$ & - & - \\
\hline Durchgehende linksseitige Spalte & - & 4 & $-7^{*}$ & - & - \\
\hline
\end{tabular}


Das functionelle Resultat bei den 30 Operirten, wo ein vollständiger Verschluss der Spalte erreicht werden konnte ist, wie folgt: Der Kranke, der nur eine Spalte im harten Gaumen hatte, bekam durch den Schluss der Spalte eine normale Sprache.

Ueber das weitere Schicksal von 2 Kranken mit Spaltung des weichen Gaumens konnte Näheres nicht in Erfahrung gebracht werden. Der Letztoperirte hat ohne Sprechunterricht schon jetzt eine nahezu normale Sprache und soll nächstens systematischen Sprechunterricht bekommen. Ein ideales Resultat steht in Aussicht.

2 der Operirten mit Spaltung des weichen Gaumens und der horizontalen Platte des Gaumenbeins nahmen bis heute keinen Sprechunterricht. Die Sprache ist bei Beiden verständlich, aber stark nasal.

18 Kranke wurden nach der Operation mit B rugger'schem Obturator versehen und nahmen Sprechunterricht, der von 17 Tagen im Minimum bis zu 12 Wochen im Maximum dauerte. Die durchschnittliche Dauer des Sprechunterrichts beträgt bei diesen 18 Kranken 6 Wochen. Von 8 dieser Kranken ist mir bekannt, dass sie den Obturator später ohne Nachtheil für die Sprache weglegten. Die Resultate dieses Sprechunterrichts plus Rachenobturator sind 12 mal die Erzielung einer normalen Sprache, 2 mal einer fast normalen Sprache, 4 mal wurde die Sprache bedeutend besser, d. h. sie wurde gut verständlich, blieb aber in mässigem Grade nasal.

6 der Operirten nahmen Sprechunterricht ohne Zuhilfenahme eines Obturators, und zwar 2 mal 2 Wochen, 1 mal 3, 1 mal 4, 1 mal 5 und 2 mal 12 Wochen. Alter der Kranken 2 mal 16 Jahre, 21, 22 24 und 40 Jahre. In 5 Fällen wird die Sprache normal und nur in einem Fall, bei einem 22jährigen Schneider, bleibt sie leicht nasal. Bei der 40 jährigen Kranken mit einer Spalte, die durch den weichen Gaumen bis zur Mitte des Process. palatin. des Oberkiefers ging und bei der es sich um eine ungewöhnlich breite Spalte (im harten Gaumen $1 \frac{1}{2} \mathrm{~cm}$, an der Grenze des harten und weichen Ganmens $2 \mathrm{~cm}$, in der Mitte des weichen Gaumens $21 / 2 \mathrm{~cm}$ ) handelte, wurde, obwohl die Kranke mit Hiobsgeduld 5 Jahre lang den schweren Suersenschen Obturationspflock getragen hatte, durch 5 wöchentlichen Unterricht eine normale Sprache erzielt!

Es folgt anbei noch eine Gesammttabelle aller 82 angeborenen Gaumenspalten mit Rücksicht auf die Beschaffenheit der Spalte und das erreichte operative Resultat (Tabelle I) und eine tabellarische Zusammenstellung der functionellen Resultate der 71 mit vollem Erfolg operirten Gaumenspalten (Tabelle II). 
Tabelle I.

\begin{tabular}{|c|c|c|c|c|c|c|}
\hline \multirow[b]{2}{*}{ Art der Spalte } & \multicolumn{2}{|c|}{$\begin{array}{c}\text { Vollkommener Verschluss } \\
\text { der Spalte }\end{array}$} & \multirow{2}{*}{ 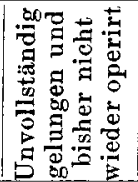 } & \multirow{2}{*}{ 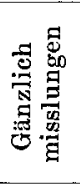 } & \multirow{2}{*}{ 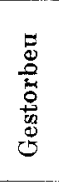 } & \multirow{2}{*}{$\underset{\Xi}{\Xi}$} \\
\hline & $\begin{array}{l}\text { durch eine } \\
\text { einzige } \\
\text { Operation }\end{array}$ & $\begin{array}{l}\text { mit Hilfe } \\
\text { von Nach- } \\
\text { operationen }\end{array}$ & & & & \\
\hline $\begin{array}{l}\text { Längsovaler Defect im harten } \\
\text { Gaumen . . . . . }\end{array}$ & 1 & - & - & - & 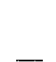 & 1 \\
\hline $\begin{array}{l}\text { Spaltung des weichen Gau- } \\
\text { mens } . . \\
.\end{array}$ & 13 & 3 & 1 & 1 & - & 18 \\
\hline $\begin{array}{l}\text { Spaltung des weichen Gau- } \\
\text { mens und der horizontalen } \\
\text { Platte des Gaumenbeins }\end{array}$ & 16 & 3 & 2 & - & 一 & 21 \\
\hline $\begin{array}{l}\text { Spaltung des Gaumens bis } \\
\text { zur Mitte des Process. } \\
\text { palatin. des Oberkiefers. }\end{array}$ & 4 & 5 & - & - & - & 9 \\
\hline $\begin{array}{l}\text { Spaltung des ganzen Gau- } \\
\text { mens mit Ausnahme des } \\
\text { Alveolarfortsatzes... }\end{array}$ & 2 & 3 & 1 & 1 & - & 7 \\
\hline $\begin{array}{l}\text { Durchgebende rechtsseitige } \\
\text { Spalte... . . . . }\end{array}$ & 3 & 4 & 1 & - & - & 8 \\
\hline $\begin{array}{l}\text { Durchgehende linksseitige } \\
\text { Spalte . . . . . . }\end{array}$ & 3 & 10 & 2 & 1 & - & 16 \\
\hline Bilateralspalte . . . . . & - & 1 & - & 1 & - & 2 \\
\hline Summa & 42 & 29 & 7 & 4 & - & 82 \\
\hline
\end{tabular}

Tabeine II.

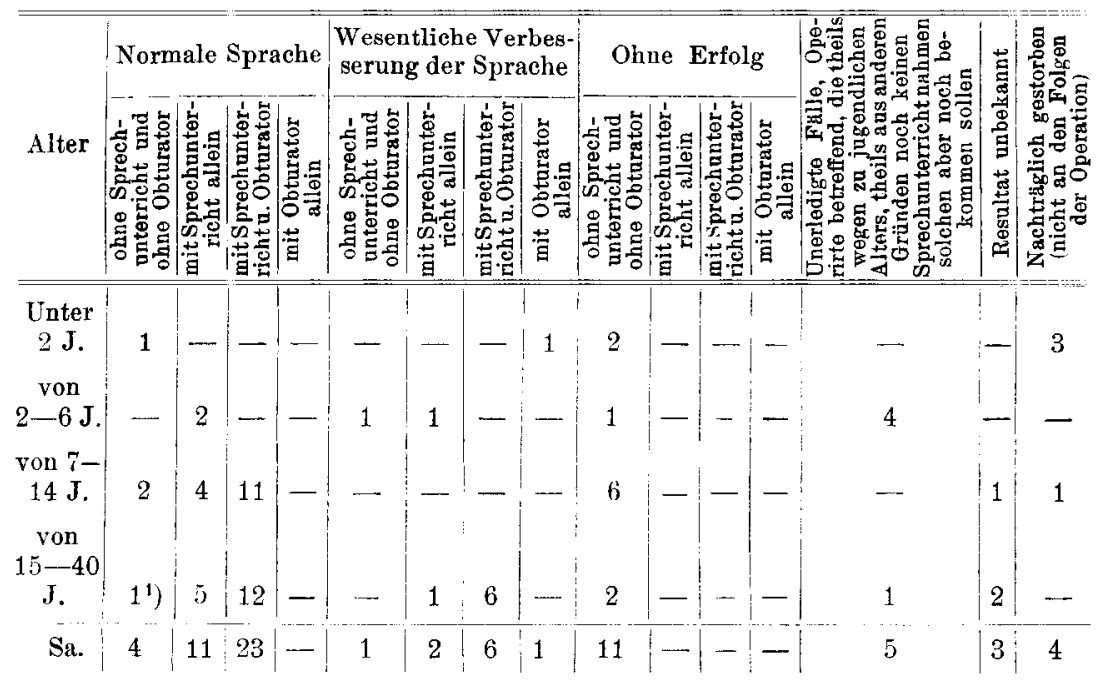

1) Hatte nur eine angeborene Spalte des harten Gaumens. 
Aus Tabelle I geht hervor, dass die Operation der Gaumenspalte bezüglich des operativen Erfolges eine ausserordentlich dankbare ist. Von sämmtlichen Operirten sind 42, d. h. 51,2 Proc., durch eine einzige Operation, 29, d. h. 35,3 Proc. durch wiederholte Operationen geheilt worden, es ist ein vollständiger Erfolg erreicht worden bei 71 Operirten, $d$. h. bei 86,5 Proc. Unvollständig gelungen sind 7 Operationen, d. h. 8,5 Proc., und gänzlich misslungen sind 4 Operationen, d. h. 4,8 Proc., und es ist nicht ausgeschlossen, dass auch bei diesen 11 unvollkommen und gänzlich ungeheilten Kranken durch weitere Operationen ein schliesslich befriedigender Erfolg zu erreichen wäre. An den unmittelbaren Folgen der Operation ist kein Patient gestorben.

Die Tabelle II lehrt wie folgt: Von den 71 glücklich operirten Kranken sind bis heute 4 gestorben, bei 3 ist das functionelle Resultat unbekannt, es bleiben also noch 64 verwerthbare Fälle. Von diesen bekamen 38 (59,3 Proc.) theils ohne weitere Behandlung, theils mit Sprechunterricht allein, theils mit Sprechunterricht plus Obturator eine normale Sprache, 10 d. b. 17,1 Proc. eine wesentliche Besserung der Sprache (sie wurde deutlich verständlich, behielt aber nasalen Beiklang), 5 der Kranken harren noch des Sprechunterrichts, 11, die niemals Sprechunterricht nahmen, verbesserten durch die Operation ihre Sprache nicht wesentlich.

Es geht daraus deutlich hervor, dass nach einer gelungenen Operation die anatomischen und physiologischen Verhältnisse des Gaumens und des Rachens so liegen, dass in der Mehrzahl der Fälle durch Sprechunterricht oder durch Sprechunterricht mit Zuhilfenahme eines Rachenobturators ein für eine normale Sprache hinreichender Abschluss zwischen Mund-und Nasenhöhle herbeigeführt werden kann, dass aber die Operation allein nur in ganz seltenen Fällen einen vol. len functionellen Erfolg, d. h. eine normale Sprache, mit sich führt.

Nur 3 von meinen glücklich operirten Kranken, einer der Altersperiode unter 2 Jahren, 2 der Altersperiode von 6-14 Jahren angehörig, bekamen ohne weiteres Dazuthun eine normale Sprache. Damit komme ich zu der vielumstrittenen Frage, in welchem Alter die angeborene Gaumenspalte bei freier Wahl operirt werden soll.

Während früher die Operationsversuche bei Kindern frühesten Lebensalters grösstentheils misslangen und deshalb wieder aufgegeben wurden, haben die Fortschritte in der Technik der Uranostaphylorrhaphie. die wir zumeist dem Vorgehen J. Wolff's verdanken, auch 
der Operation in diesem Lebensalter eine grössere Sicherheit des Erfolges gegeben. So Lat J. Wolff (1. c.) in den Jahren 1872-1900 bei 128 Kindern im Alter von 1-35 Monaten 67 Gaumenspalten durch eine einzige Operation zum Schluss gebracht, 30 mal mit Hilfe von Nachoperationen, während 31 misslangen oder an den Folgen der Operation starben. Ehrmann ${ }^{1}$ ) hatte bei 10 Kindern unter 2 Jahren 6 mal vollen Erfolg. Ich erreichte bei 7 unter 12 Kindern im Alter unter 2 Jahren die vollständige Vereinigung der Spalte, 5 mal durch eine Operation, 2 mal mit Hilfe von Nachoperationen. Aber wenn auch aus diesen Thatsachen hervorgeht, dass die Frühoperation heute bessere Chancen bietet, als zur Zeit der ersten Versuche durch Langenbeck, Billroth, Otto Weber, Gustar Simon u.s.w., so ist eben doch die Zahl der Misserfolge noch immer eine verhältnissmässig grosse. So hat $\mathrm{W}_{0}$ lff bei Kindern von $1-35$ Monaten unter 128 Operationen 19 Misserfolge resp. unvollständig gelungene und misslungene Operationen, d. h. 14,8 Proc.; bei Kindern von 3 bis 6 Jahren dagegen auf 64 Operationen nur 5 Misserfolge, d. h. nur 7,8 Proc.

Ehrmann zählt unter 10 Operationen bei Kindern unter zwei Jahren 2 Misserfolge, während er bei 20 Operationen bei Kindern von 2-6 Jahren nur einen Misserfolg erlebte. lch selbst hatte bei 12 Operationen von Kindern unter 2 Jahren 2 vollständige Misserfolge und 3 theilweise Erfolge, während auf 11 Operationen an Kindern von 2-6 Jahren 2 Misserfolge und keine unvollständig gelungene Operationen kommen.

Von 2 Kindern der v. Bergmann'schen Klinik im Alter von $11 / 1-11 / 2$ Jaliren wurde das eine geheilt, das andere fast ohne Erfolg operirt, indem in der Mitte sämmtliche Nähte durchschnitten und der Spalt in seiner alten Breite klaffte. ${ }^{2}$ )

Als grossen Gewinn der frühzeitigen Operation hebt J. Wolff die lebensrettende Bedeutung der gegliickten Operation hervor. Es ist bekannt, dass Kinder mit Gaumenspalten grosse Neigung zu Respirations- und Digestionskrankheiten haben, dass die Ernährung dieser Kinder oft Schwierigkeiten macht und dass sie, schon elend zur Welt gekommen, in Folge mangelhafter Ernährung leicht zu Grunde gehen. $W_{0}$ lff hat auch durch seine Körpergewichtsmessungen den günstigen Erfolg der geglückten Operation auf die Ernährung nachzuweisen gesucht. Dabei ist aber nicht zu vergessen, dass die Operation in einem

1) J. Ehrmann de Mulhouse, Des opérations plastiques sur le palais chez l'enfant. Paris 1899.

2) Ueber Gaumenspalten. Inaug.-Diss. von L. D ob b e rkau. Berlin, Ebering. 
sehr frühen Alter an und für sich durchaus nicht ungefährlich ist. J. Wolff zählt unter 128 Operationen bei Kindern im Alter von 1 bis 35 Monaten 12 Todesfälle (9,3 Proc.), dagegen unter 64 Operationen an Kindern von 3-6 Jahren nur 2 (3,1 Proc.). Ehrmann erlebte bei 10 Operationen an Kindern von weniger als 2 Jahren 2 Todesfälle und Lawson Tait I) beklagt unter 5 Operationen bei Kindern unter 2 Jahren einen Todesfall als Folge der Operation.

Nicht ausser Acht zu lassen ist ferner bei der Frage der lebensrettenden Bedeutung der frühzeitigen Operation, dass von den glücklich operirten Kindern später noch auffallend viele im frühen Kindesalter zu Grunde gehen. Von den 6 geheilten Kindern Ehrmann's ist nach 4 Jahren nur noch eines am Leben, eines starb 3 Monate nach der Operation an Variola, eines 2 Jahre später an Pneumonie und 3 an tuberculöser Meningitis 2, 3 und \pm Jahre später. Von meinen 7 glücklich operirten Kindern ist eines 3 Wochen später einem Erysipel erlegen, ein zweites 1/4 Jahr später an Lungenentzündung, ein drittes 10 Monate nach der Operation an Meningitis und ein viertes 9 Jahre später an Diphtherie gestorben, während von den 11 in der Altersperiode von 2-6 Jahren operirten Kindern heute noch alle am Leben sind. Es scheint daraus hervorzugehen, dass der Schutz der frühzeitigen Operation gegen schädliche Einflüsse, besonders gegen Infectionskrankheiten und auch gegen Lungenaffectionen, nur ein sehr relativer ist.

Es bleibt noch der weitere Gewinn der frühzeitigen Operation, den Wolff ihr nachrïhmt, dass bei derselben in der Regel ohne Zuhilfenahme von Sprechunterricht oder Prothese ein voller functioneller Erfolg, d. h. eine normale Sprache, erreicht werde, zu prüfen übrìg und ich bemerke gleich, dass auch ich der Frühoperation den unbedingten Vorzug gäbe, wenn sie fast ausnahmslos den vollen functionellen Erfolg gewährleistete.

Wie verhält es sich nun damit? Sehen wir zunächst nach den Erfahrungen J. Wolff's. In seiner Abhandlung über die frübzeitige Operation der Gaumenspalte berichtet er über einen Fall eines 7jährigen Knaben mit einer Gaumenspalte bis nahe an den Alveolarrand, der im Alter von 6 Monaten zweizeitig operirt wurde und der, ohne besonderen Sprechunterricht zu haben, absolut normal spricht, sodann von einem 6 jährigen Mädchen, das im Alter von $1 \frac{1}{1} 2$ Jaliren wegen einer ebenfalls bis nahe an den Alveolarrand reichenden Spalte operirt worden war. Auch hier war die Sprache ideal rein, und da von

1) The British and Foreign med. chirurg. Review. July 1870. p. 181. 
Sprechunterricht nichts gesagt ist, muss wohl angenommen werden, es habe keinen solchen gehabt. Dann erinnert er an 3 frïher vorgestellte Fälle nicht durchgehender Spalten, an einen 7 Jahre alten, im 13. Monat operirten Knaben, der nach 2 monatlichem Sprechunterricht bei Gutzmann fast alle besonders schwierigen Worte vollkommen deutlich ohne nüselnden Beiklang und ohne Verziehung der Gesichtsmuskeln aussprach; an einen 6 Jahre alten, im Alter von 15 Monaten operirten Knaben mit vollkommen idealem Spracherfolg nach 2 Monate hindurch genossenem Sprechunterricht; an einen 9 jährigen, im Alter von 14 Monaten operirten Kranken, der, obwohl er niemals Sprechunterricht genossen batte, eine normale Sprache erlangt hatte; an ein im Alter von 4 Monaten operirtes Mädchen, dessen Sprache 2 Jahre später eine so befriedigende war, dass Niemand auf ihr ehemaliges Leiden aufmerksam wurde, der nicht ohnehin davon wusste. Und endlich erwähnt er eines 51/2 Jahre alten Kindes, das im Alter von 1 Jahr und 8 Monaten operirt, fast absolut tadellos spricht.

Anderweitige Mittheilungen W olff's über die functionellen Erfolge der Gaumenspaltenoperation bei Kindern unter 2 Jahren konnte ich nirgends finden.

Nun hat aber J. Wolff nach Tabelle I 112 Kinder $1 \mathrm{~m}$ Alter von 1-23 Monaten operirt. Aus dem Erfolg in diesen 6 Fällen von Frühoperation bei Kindern, von denen 3 eine ideale Sprache ohne besonderen Sprechunterricht, eines eine sehr befriedigende und 2 wiederum eine normale Sprache erst nach zweimonatlichem Sprechunterricht erlangten, ist doch wohl kaum der Schluss zu ziehen, dass bei der im frühesten Kindesalter unternommenen Operation die normale Sprache ohne jedes Hinzuthun mit Sprechunterricht oder Prothese die Regel sei.

Billrothi) beobachtete bei einem im Alter von 11 Monaten glïcklich operirten Kinde mit doppelter Lippen- und durchgehender linksseitiger Gaumenspalte nach Ablauf von 4 Jahren, dass dasselbe mit entschiedenem Gutturalton sprach. Bezüglich meiner Resultate will ich nur kurz recapituliren. Von den 3 Kindern, die im Alter unter 2 Jahren operirt und später wieder untersucht wurden, bleibt bei zweien die Sprache ohne Unterricht näselnd und undeutlich, während eines ohne weiteres Hinzuthun für den Laien normal und für den Sprachlehrer fast normal spricht.

1) Th. Billroth, Gesammtbericht über die chirurgischen Kliniken in Zürich Imd Wien. Berlin 1879. 
Ueberdies ist wohl zu berücksichtigen, dass die guten und idealen functionellen Resultate durchaus nicht etwa nur dem Alter von unter 2 Jahren eigenthümlich sind. Ich operirte einen Knaben von 11 Jahren und ein Mädchen von 14 Jahren, die (s. oben) ohne Sprechunterricht und ohne Obturator zu einer normalen Sprache gelangten. Schede 1) erzählt sogar von 2 Erwachsenen, die unmittelbar nach vollendeter Heilung der Gaumenspaltenoperation in kurzer Zeitabsolut gut sprachen.

Wichtiger als die eben erwähnten und bei ihrer geringen Zahl noch keineswegs ausschlaggebenden, günstigen, functionellen Resultate der Gaumenspaltenoperation bei Kindern unter 2 Jahren scheint mir 1. die rein theoretische Erwägung, dass je früher ein genähter Gaumen als Ganzes beim Schlucken, Husten, Niesen u. s. w. in Action tritt, desto kräftiger er auch für seine Functionen beim Sprechen wird und 2. die Erfahrung der mit Gaumenspalten sich beschäftigenden Sprachlehrer, wonach man nach frühzeitigen Operationen bei den operirten Kindern später fast nur oder nur reine Laute findet. Der Mangel an Fertigkeit in der Verbindung derselben ist gering und wird durch Uebung bald ausgeglichen. Das gilt aber nicht nur für Kinder unter 2 Jahren, das gilt auch noch für Kinder von 2-6 Jahren, immer aber unter der Voraussetzung, dass überhaupt der weiche Gaumen resp. seine Hälften so reich sind, dass nach der gelungenen Naht ein Verschluss der Nasenhöhle ohne Obturator möglich ist.

Ich meine daher, man sollte in einem Lebensalter operiren, wo die Mortalität der Operation gleich Null oder nahezu Null ist, wo ein idealer functioneller Erfolg (bezüglich der Sprache) ohne weiteres Hinzuthun möglich und mit Sprechunterricht fast in allen Fällen sicher ist, wo die Kinder noch nicht in die Schule gehen, d. h. noch nicht in's Leben mit seinen Anfechtungen und Kränkungen herausgetreten sind. Das wäre die Zeit vom 2.-6. Lebensjahre, wobei dann jeder Operateur sich je nach Constitution, Temperament, Ernährungszustand des Patienten und nach seinen eigenen Operationserfolgen die nähere Zeitbestimmung der Operation selbst aussuchen kann. -

Meine ersten Uranostaphylorrhaphien unterschieden sich in keiner Weise von der ursprünglichen v. Langenbeck'schen Plastik. Später adaptirte ich die mannigfachen wirklichen Verbesserungen, die die

1) Verhandlungen der deutschen Gesellschaft für Chirurgie.

2) Predöhl, Zur operativen Behandlung der Gaumenspalten. Jahrbücher der Hamburgischen Staatskrankenanstalten. 1. Bd. 1889. 
Operation namentlich durch J. Wolff erfuhr, und verfahre schon seit Jahren in folgender Weise:

Neben den üblichen Vorbereitungen bekommt der Kranke einen oder mehrere Tage vor der Operation Mund- und Nasenspülungen mit 1 proc. Salicyllösung. In der Regel wird chloroformirt, doch machte ich einige Male die ganze Operation unter Cocainisirung der Schleimliaut ohne Chloroform, zuletzt bei einem 21 jährigen Gärtner, der sich wegen eines Herzleidens nicht narkotisiren lassen wollte. Er hielt auch bei der ausserordentlich schmerzhaften Ablösung des mucösperiostalen Gaumenlappens musterhaft und ohne sich zu rühren Stand. Bei einigen schwächlichen Kindern und Erwachsenen beschränkte ich die Chloroformirung auf den Act der Loslösung des mucösperiostalen Gaumenüberzugs und machte sowohl Anfrischung als Naht ohne Chloroform.

Ist der Kranke chloroformirt, so wird er in Rose'sche Kopflage gebracht, nachdem das Whitehead'sche Speculum - das allein oder noch besser in Verbindung mit den v. Langen beck'schen Mundwinkelhaltern die Operation ausserordentlich erleichtert und das in verschiedenen Grössen vorhanden sein muss - eingelegt worden ist. Der Kopf wird durch einen hierauf eingeübten Assistenten gestützt. Trotz sorgfältiger Anlegung des Whitehead'schen Speculums geschieht es mitunter, dass im Verlaufe der Operation die Zunge nach binten rutscht und die Epiglottis auf die Rima drüekt, wobei asphyktische Symptome auftreten, denen man leicht dadurch begegnen kann, dass man die Zunge anschlingt und unter dem Zungenspatel nach vorne zieht.

Als ersten und schwierigsten Act der Operation vollführe ich von den Langenbeck'schen Seitenschnitten aus die Loslösung der mucösperiostalen Gaumenlappen. Ich hatte einige Male das Missgeschick, dass die schon zum Theil abgelösten Lappen bei einer raschen, unerwarteten Bewegung des Kopfes seitens des erwachenden Patienten durch Ausgleiten des Elevatoriums seitliche Einrisse bekamen. Stets führte ich mit doppelter Vorsicht die Ablösung zu Ende, nähte dann sofort die Einrisse und sah dann niemals eine Schädigung der Lappen. Die Blutung ist schon bei Anlegung der Seitenschnitte und auch bei Ablösung der Lappen oft sehr erheblich, kann aber durch Compression mit Finger und kleinen Tampons - ich nehme dazu frisch ausgekochte Schwämme - auf ein Minimum beschränkt werden.

Um die Entspannungsschnitte im Velum zu vermeiden, machte ich theils die stumpfe Abtrennung des Involucrum palati duri von den den Partes horizont. zunächst angrenzenden Partien des Process. 
pyramidalis des Gaumenbeins, theils nach Billroth die Abmeisselung der medialen Platte des Process. pterygoid. ossis sphenoid. und deren Dislocirung nach innen, die v. Eiselsbergi) für das beste Verfahren hält. Es sei gleich hier erwähnt, dass in den 6 Fällen, in denen ich das Billroth'sche Verfahren anwandte, das functionelle Resultat 4 mal ein vorzügliches war. $\mathrm{Zu}$ dem zweiten Act, der Anfrischung der Lappen, bediene ich mich eines scharfen Häkchens, mit dem die betreffende Velumhälfte ungefähr $1 \mathrm{~cm}$ vor der Uvulaspitze angehakt wird, und eines Beer'schen Staarmessers, mit dem zuerst der Uvulatheil und nachher der vordere Theil des Lappens breit angefrischt wird.

Für den dritten Act, die Naht, verwende ich entweder nur kleine krumme Nähnadeln, die mit dem gewöhnlichen Nadelhalter eingeführt werden, oder dann die Fer gusson'schen gestielten Nadeln, durch die auf der einen Seite in bekannter Weise die Schlinge, auf der andern der Faden eingeführt und mit Hilfe der Schlinge durch den andern Wundrand gezogen wird. Bei Erwachsenen brauche ich mehr die Fergusson'schen, bei Kindern mehr die gewöhnlichen krummen Nadeln. Das Nähmaterial ist Seide, Silberdraht und Aluminiumbroncedraht. Als eine Neuerung von Bedeutung halte ich die Wollf'sche Naht der hinteren Fläche der Uvula und der Arcus pharyngo palatini. Diese hinteren Nähte geben der ganzen Naht mehr Festigkeit und Sicherheit, nur muss man nicht zu eng nähen, sonst riskirt man theilweise oder vollständige Gangrän der Uvula, wie ich es zweimal erlebte.

In die Seitenschnitte legte ich früher regelmässig kleine Jodoformgazemèchen, die 3-4 Tage liegen blieben. Auf Grund einiger Erfahrungen bin ich davon abgekommen und überlasse die Seitenschnitte ihrem Schicksal. Ich sah einmal bei einem Erwachsenen in Folge dieser Jodoformgazeeinlagen eine ausserordentlich reichliche und lästige Schleim- und Speichelabsonderung, Krusten- und Borkenbildung an der Schleimhaut des Gaumens, so dass dadurch das operative Resultat tagelang gefährdet war; wiederholt aber sah ich hartnäckige und den Appetit raubende Catarrhe der Mund- und Nasenschleimhaut. Das Weglassen der Jodoformtampons hatte keine üblen Folgen und beförderte die rasche Heilung.

J. Wolff hat bei Kindern unter 3 Jahren das zwei- und dreizeitige Operationsverfahren eingeführt; er löst in der ersten Sitzung beide oder auch nur einen mucösperiostalen Lappen und nimmt dann

1) v. Eiselsberg, Zur Technik der Uranoplastik. Archiv f. klin. Chirurgic. 64. Bd. Heft 3 . 
erst 5-8 Tage später unter nochmaliger Lösung des oder der Lappen die Wundmachung und Vernähung der Spaltränder vor. Ich besitze keine eigene Erfahrung über diese Modification der Uranostaphylorrhaphie (ich machte sie ein einziges Mal ohne Erfolg) und vollende die Operation auch der breitesten Spalten in einer Sitzung, denn es widerstrebt mir, eine Operation, die in einer Sitzung vollendet werden kann, in 2 und 3 Abtheilungen zu zerlegen und die Kranken einer zwei und dreifachen Operation und Narkose auszusetzen.

Wolff will durch die zweizeitige Operation die Entstehung zuweilen auftretender Randnekrosen bei primär verheilten Lappen verhüten und das Klaffen der Seitenschnitte, das seiner Ansicht nach leicht zu einer Wundinfection führen kann. Ich sah bei meinen 82 Fällen niemals eine von den klaffenden Seitenschnitten ausgehende Wundinfection.

Ueble Zufälle und Complicationen des Wundverlaufs sind, wenn man von der totalen oder partiellen Gangrän der Wundränder und consecutiver Bildung von mehr weniger grossen Spalten und Löchern, die sich von selbst schliessen oder Nachoperationen nach dem Typus der totalen Uranostaphylorrhaphie verlangen, absieht, recht selten.

Fieber kommt nicht häufig vor und beschränkt sich fast immer auf 2-4 Tage, am häufigsten sahen wir fieberhaften Verlauf bei Kindern unter 2 Jahren. Im Ganzen nur 6 mal begegneten wir am 1. Operationstage stärkeren Nachblutungen aus den Seitenschnitten, die jeweils prompt durch Fingercompression gestillt werden konnten.

Lateralspalten, d. h. Fisteln zwischen den Seitenschnitten und dem Nasenrachenraum, denen $\mathrm{Eh} \mathrm{r}^{\mathrm{m}} \mathrm{ann}^{1}{ }^{1}$ ) eine besondere und interessante Abhandlung widmete, erlebten wir nur 2 mal, einmal bei einem 6 jährigen Mädchen, wo sich 7 Tage nach der Operation ein 5 und $4 \mathrm{~mm}$ breites, ovales Loch zwischen dem linken Seitenschnitt und dem Nasenrachenraum gebildet hatte, das sich nach weiteren 14 Tagen von selbst schloss, und dann bei einem 8 jährigen Knaben, wo sich durch Aufrollen des rechten mucösperiostalen Seitenlappens auf der Höhe des 1. Backenzahns ein ovales Loch von $5 \mathrm{~mm}$ Länge zwischen rechtem Seitenschnitt und Nasenhöhle gebildet hatte, das durch einen kleinen Lappen noch nicht benutzten mucösperiostalen Ueberzugs plastisch geschlossen werden konnte.

Bei einer 19jährigen Kranken mit ausserordentlich breiter Spalte bis zum Alveolarfortsatz (an der Grenze zwischen hartem und weichem Gaumen war sie $21 / 2 \mathrm{~cm}$ breit) und sehr schmalen Processus palatini

1) Ehrmann, Sur les fistules latérales du palais consécutives à l'Uranostaphylorthaphie. Paris 1897. 
der Oberkieferhälften gelang bei der ersten Operation nur die Vereinigung des weichen Gaumens, während die Naht des harten Gaulmens wieder auseinander ging. Daher $1 \frac{1 / 4}{4}$ Jahr später 2. Uranoplastik, wobei am rechten mucösperiostalen Lappen die Verbindungsbrücke mit dem weichen Gaumen sehr schmal ausfiel. Am 3. Tage nach der Operation hatte sich der rechte Lappen hinten vom weichen Gaumen getrennt, und während nun die Vereinigung der angeborenen Längsspalte auch im harten Gaumen glücklich zu Stande kam, hatte sich jetzt eine neue transversale Spalte zwischen rechtem harten und weichen Gaumen gebildet. Die Gaumenplatte, an dem der nachher angelegte Brugger'sche Rachenobturator befestigt war, deckte auch diese Spalte und die mit dem so erhaltenen Resultat ganz befriedigte Patientin konnte sich bis jetzt zu einer Nachoperation nicht verstehen.

Zurückbleiben des Wachsthums des Oberkiefers beobachteten wir nur 2 mal, bei einem im 14. Lebensjahr operirten Knaben mit durchgehender rechtsseitiger Gaumenspalte, wo beim Kieferschluss die Zähne des Unterkiefers die des Oberkiefers überragten, was dem Gesicht einen verkniffenen Ausdruck gab und bei einem mit $3 \frac{1}{2}$ Jahren operirten Mädchen, das mit 15 Jahren nachuntersucht wurde und wo beim Kieferschluss die Bisskanten der obern und untern Schneidezähne direct aufeinander stiessen.

Bezüglich der Nachbehandlung halte ich die von J. Wolff angerathenen und später wieder verlassenen Irrigationen der Mund- und Nasenhöhle mit Salicylwasser für sehr nützlich bei Erwachsenen und grösseren Kindern, selbstverständlich in aufrechter Stellung und ohne Narkose. Sie sind den Kranken sehr angenehm, erleichtern die Athmung, indem sie die mit Schleim- und Blutborken verlegten Nasengänge frei machen und durch Beseitigung des Mundsecretes und Reinigung der Zunge den Appetit fördern. Im Uebrigen gestatten wir schon vom 1. Tage an flüssige Nahrung und gehen nach Entfernung der Nähte zu Brei und Fleischmuss über.

Ist die Wunde solid vernarbt, so wenden wir sofort regelmässig. Massage des neugebildeten weichen Gaumens an. Mit dem geölten Zeigefinger wird das Velum von einer Tonsille bis zur andern kräftig gestrichen und gegen die Choanen gedrängt. Diese Massage wird auch wähernd des Sprechunterrichts fortgesetzt. Ich berichtete über diese Massage schon im Jahre 1889 in der Naturforscherversammlung zu Heidelberg. Später las ich, dass v. W in i warter schon im Jahe 1885 im Chirurgencongress eine ähnliche Massagebehandlung des operirten Gaumens empfahl.

Zur Herbeiführung eines raschen Schlusses von kleinen, nach der 
Operation zurückgebliebenen Spalten oder Löchern haben uns die K ü s t e rschen Bepinselungen mit Cantharidentinetur gute Dienste geleistet.

Da nun die Fälle, wo nach vollständig gelungener Operation die Sprache ohne weiteres Hinzuthun eine reine und normale wird, wie oben auseinander gesetzt und statistisch festgelegt wurde, ausserordentlich selten sind, und alle Versuche, durch besondere Operationsverfahren das Zustandekommen des Gaumenklappenschlusses in jedem Falle zu erzwingen, bis jetzt erfolglos blieben, so haben wir zur Herstellung einer normalen Sprache nach gelungener Operation nur ein Mittel: den Sprechunterricht, eventuell mit Hilfe eines Rachenobturators.

Wenn man daher an seinen Gaumenspaltenoperationen wirkliche Freude und Genugthuung erleben soll, so muss man einen tüchtigen Sprachlehrer und einen geschickten Zahnarzt zur Seite haben. Ich hatte nun das Glück, in den Herrn Schittenmüller und Wang in Meersburg zwei Sprachlehrer zu finden, die mit bewundrungswerther Geduld und Ausdauer und mit der nöthigen Sachkenntniss, die ihnen ihre Thätigkeit an der Grossh. Taubstummenanstalt verschaffte, zugleich aber auch mit der grössten Uneigennützigkeit gegenüber Unbemittelten, den anstrengenden Sprechunterricht ertheilten. Ihre Erfolge sind um so beachtenswerther, da die Dauer des Unterrichts im Grossen und Ganzen eine verhältnissmässig kurze ist und im Durchschnitt nur 6 Wochen betrug. Die $\Lambda$ rt und Weise des Unterrichts ist in der beifolgenden Zuschrift der Herren $W$ ang $g$ und Schittenm üller skizzirt.

Ueber die Sprache der Patienten mit operirtem angeborenen Gaumendefect bei ihrem Einiritt in unsere Behandlung ist Folgendes zu sagen:

Der Gesammteindruck der Sprache ist nasal. Von den Vocalen ist a relativ der reinste; das Nasale steigert sich bei denselben, je weiter sie in der Reihe $a, o, e, u, i$ zurückstehen. Die Tenues $p, t, k$, wie auch die Mediae b, d, g werden mit Durehschlag der Luft durch die Nase gelbildet. Ilr Explosionsgeräusch entstelit nicht an der Articulationsstelle, sondern wird im Kehlkopf erzeugt, und zwar dureh explosionsartige Sprengung des Stimmritzenverschlusses in Moment der Articulation. Bei den Spiranten ist der Durchschlag dureh die Nase sehr stark; daher findet man hier durchgehends ein schnaulvendes Geräusch in der Nase, das jedoch von manchen Patienten dureh Engenbildungen in der Nase mittelst Bewegungen der Nasenflügel so gesehickt modificirt wird, dass man richtig gebildete Laute zu hören vermeint. ch ist fast ausschliesslich in der a-Lage vorhanden, lautet raul, kurz und nicht rein; h wird meist seicht gegeben, verhältnissmässig gut erscheint manchmal sch. Zungen- $\mathrm{r}$ fehlt immer, und weil das Gaumen-r des fehlenden Zäpfehens wegen nicht möglich war, so ersetzt der Patient dasselbe durch ein Kratzen in der Rachenhöhle unter verstärkender Zugabe von Speichelgeräuseh. j, 1 und w haben nasalen Klang; w wird nur flüchtig angedeutet. Die Resonanten $m$ und $n$ sind natürlich 
richtig; ng fehlt fast immer und wird gewölinlich durch n ersetzt. Die angeführten Articulationsfehler treten bei dem Einen stärker und allgemeiner in die Erseheinung, als bei dem Andern, je nach der Art des Defects und nach der Geschicklichkeit im Imitiren einzelner Consonanten; dadurch werden die versehiedenen Grade der Verständlichkeit der Sprache bedingt.

Der beim Sprechen verwendete Luftstrom ist seicht, der Redefluss häufig durch zu rasch sich wiederholendes Einathmen zerhackt, es fehlt an rationeller Athmungsökonomie. Das Gesicht zeigt oft Verzerrungen, die Lippen sind gewöhnlich blass, von unkräftiger Musculatur und wenig modellirfähig; beim seitherigen Sprechen wurden sie eben nur wenig in Anspruch genommen. Die Zunge hat ihre Ruhelage nach hinten; ihr Rücken ist gewöhnlich stark gewölbt; ihr vorderer Theil erweist sich bei befohlenen Bewegungen sehr unsicher und schwach, da sich die Zungenspitze bislang beinahe ganz passiv verhielt. Nicht selten findet man bei Fällen mit gespaltener Oberlippe die Zunge einseitig entwickelt. Dem operirten weichen Gaumen fehlt es noch an Elasticität und entsprechender Energie in seinen Bewegungen. Das Gehör der Patienten zeigt sich ihren Sprachfehlern gegenüber selir abgestumpft, ein Umstand, der die Arbeit oft ausserordentlich ersehwert.

Da die bezeichneten Sprachfehler mit ihren sämmtlichen Nebenerscheinungen ihren letzten Grund in der bedingten und angewöhnten abnormen Athmungsthätigkeit haben, setzt der Unterricht hier ein mit folgenden Athmungsübungen:

1. Langes, ruhiges und tiefes Ein- und Ausathmen durch den Mund hezw. durch die Nase.

2. Kurzes, tiefes Einathmen dureh den Mund und ruliges, langes Ausathmen dureh denselben.

3. Kurzes, tiefes Einathmen durch den Mund und stossweise hanchendes Ausathmen durch denselben; ebenso durch die Nase.

4. Kurzes, tiefes Einathmen durch den Mund und Ausathmen durch die Nase wie unter 2 und 3.

5. Einathmen durch den Mund, Ausathmen durch die Nase, Einathmen durch die Nase, Ausathmen durch den Mund, in rasehem Wechsel.

6. Liurzes, tiefes Einathmen durch den Mund, Versehluss der Stimmritze, stossweises Ausathmen mit jeweiliger Stimmritzenexplosion.

7. Blasen mit eontinuirlichem Luftstrom.

9. Tiefes Einathmen, Pressung der Luft in der Mundhöhle.

9. Oftmaliges stossweises Blasen in einem Ausathmungsstrom.

Die Uebungen unter 5, 7, 8 und 9 haben noch den speciellen Zweck, Verschluss und Oeffnung des Luftwegs durch die Nase mittelst des Obturators sobald als möglich in die Gewalt des Patienten zu bringen.

Die Athmungsübungen werden während der ganzen Uebungszeit fortgesetzt. Es können nicht alle sofort auftreten, sondern sie werden mit Rï̈cksicht auf die fortschreitende Kräftigung der Respirationsthätigkeit beigezogen. Beispielsweise bedarf es immer 8-10tägiger Bemühungen, um den Patienten zum ordentlichen Blasen zu bringen.

Sobald die unter 1, 2, 3 und 4 angeführten Athmungsübungen richtig ausgeführt werden, lernt der Patient die Organstellnngen der Vocale kennen und nachbillen, wobei er seine Leistungen mit den vorbildlichen des Lehrers 
vergleicht und entsprechend präcisirt; daher erfolgen die Articulationsübungen in der ersten Zeit ror einem Lautirspiegel

Hierauf wird das h in den Organstellungen der Vocale geülot und dann mit diesen verbunden

Nach Vornahme der Athmungsübung unter 6 werden die Vocale mit dem kurzen, scharfen Vocaleinsatz geübt. Durch Befühlen seiner Nasenflügel und auch derjenigen des Lehrers controlirt der Patient auf leichte und bequeme Weise die Reinheit seiner Vocale, der Spiranten $\mathbf{j}$, w und der Liquidae I, r. Solange die Nasenflügel bei der Bildung dieser Laute vibriren, sind dieselben noeh nasal. Um die Consonanten auf ihre Reinheit zu prüfen, dient ein ans Carton hergestellter kleiner 'Teller mit einer ziemlich flachen Einbuchtung, der unter die Nase gehalten und dabei leicht an die Oberlippe angedrückt wird. Bewegen sich die auf diesem Teller liegenden Papierschnitzel bei Bildung der Consonanten oder fliegen sie gar davon, so weiss der Patient, dass es gilt, weiter zu ringen. Diese Prüfungsmittel werden während der ganzen Dauer der Articulationsübungen angewendet, sie dienen zugleich der Schärfung des abgestumpften Gehörs.

Nun schreitet man zur Bildung der Consonanten. Die Articulationsstelle wird dem Patienten genau vorgezeigt und beschrieben. Seine oft sehr unsicher manöverirenden Sprachorgane werden durch den Finger des Lehrers unterstützt und beriehtigt. Bereitet ein Consonant besondere Schwierigkeiten, so wird er in seine Elemente zerlegt; diese werden einzeln so lange geübt, bis die Bildung des Lautes gelingt. Der jeweils gewonnene Consonant wird sofort mit den Vocalen zu Reihen von An- und Auslautsilben verbunden, und zwar deshalb zunächst zu Anlautsilben, weil in diesen durch den vorangehenden Voeal der für die Bildung des Consonanten nothwendige Abschluss des Nasencanals schon vollzogen ist.

Gewöhnlich werden die Consonanten in nachstehender Reihenfolge geübt:

$\mathrm{p}, \mathrm{b}, \mathrm{t}, \mathrm{d}, \mathrm{l}, \mathrm{k}, \mathrm{g}, \mathrm{seh}, \mathrm{w}, \mathrm{f}, \mathrm{ch}, \mathrm{s}, \mathrm{z}, \mathrm{x}$.

Mit den Zungen-r-Uebungen beginnen wir schon in den ersten Stunden; sie sind ein ausgezeichnetes Mittel, die Leistungsfähigkeit des weichen Gaumens zu erhöhen.

Es werden nun Silhen mit 3 und mehr Lauten, Consonantenhäufungen, Wörter und kleine Sätze geübt; daran schliessen sich Leseübungen. Das einzelne Wort, der einzelne Satz wird mit allmählich steigendem Tempo vielfach wiederholt. Wird dabei ein Laut falsch gebildet, so ist derselbe für sich allein und in dem betreffenden Worte so lange zu üben, bis sich die richtige Muskelthätigkeit sicher vollzieht. Während bisher mit mehr als gewöhnlicher Lantstärke und sehr scharfer Articulation gesprochen wurde, findet das Lesen unter normalen Verhältnissen statt.

Den Schluss bilden Uebungen im Vortrag von Gedichten und solche im freien Erzähılen. Sehr fördernd wirken auch Gesangsübungen.

Der weiche Gaumen und die operirte Oberlippe werden während des ganzen Curses einer intensiven Massage unterzogen, wodurch die günstigsten Resultate erzielt werden. Bei Fällen ohne Obturator ist der Gebrauch unseres Gaumenreizers sehr wirksam. Derselbe wird zwischen weichem Gaumen und Passavant' schem Wulst eingeführt und bewirkt hier durch Reizung der beiden Organe erhöhte zweckentsprechende Function derselben.

Dentsche Zeitschrift f. Chirurgie. LXVII. Bd. 
Nach durchschnittlich 6 Wochen ist das Ziel erreicht, der Patient erfreut sich nunmehr einer normalen Sprache, $d . h$. einer Sprache, in welcher sämmtliche Laute richtig articulirt werden und absolut rein sind $W$ as an Geläufigkeit noch alggehen sollte, erwirbt er sich bei einiger Aufmerksamkeit ohne besondere Austrengungen in kurzer Zeit zu Hause. Die Lippen sind frisch, ja blühend und sehr beweglich. Die Gesichtsmuskeln zeigen beim Sprechen die gewohnte Ruhe. Der Patient unterscheidet nun sicher zwischen richtiger und falscher Lautbildung und corrigirt sich im gegebenen Falle sofort Das bei Beginn des Curses sich einstellende häufige tiefe Gähnen hat wieder aufgehört; offenbar hat sich die Capacität der Lunge bedeutend gehoben.

Eine merkwürdige psychische Veränderung lat sich an den Patienten vollzogen. Die zuvor gedrückten, leutschenen Menschen sind glïcklich, mittheilsam und hoffnungsfroh geworden.

Noch einige Worte in Bezug auf den Gebrauch des Obturators. Unser Ziel ist in jedem Falle eine absolut normale Sprache. Wo wir nach eingehender Prüfung die Gewissheit erhalten, in nicht zu langer Zeit und ohne übermässige Anstrengung des Patienten dieses Ziel ohne Obturator erreichen zu können, da verzichten wir selbstverständlich gerne auf denselben. Wenn uns aber ein derartiges Resultat von vornherein unmöglich erscheint, so wenden wir natürlich den Apparat an. Aber auch in zweifelhaften Fällen benützen wir ihn immer. Ist hier als Endresultat eine normale Sprache ohne Obturator überhaupt möglich, so gelangen wir anf diesem Wege am sichersten und leichtesten zum Ziel. Successive Verjüngungen sind die Etappen zur Ablegung des Apparats; der Zeitpunkt hierzu tritt in der Regel erst nach mehreren Jahren ein. Die Kosten für den Obturator werden in solehen Füllen durch die ermöglichte bedeutende Abkürzung des Uelungscurses voll aufgewogen.

Ich füge diesem Berichte noch die Bemerkung bei, dass von den beiden Herren in den letzten Jahren immer häufiger der Sprechunterricht ohne Obturator versucht wird und dass eine Einschränkung in der Anwendung des Obturators gegen früher entschieden stattgefunden hat.

Diejenigen Kranken, bei denen der Sprechunterricht nicht zum Ziele einer normalen Sprache führte, wurden mit dem Brugger'schen Obturator versehen. Der Brugger'sche Obturator stellt eine Modification des Schiltsky'schen Obturators dar. Die Befestigungsweise ist die gleiche, wie bei diesem. Er hängt durch eine bewegliche Spiralfeder mit einer Gaumenplatte zusammen, die durch Klammern an den Zähnen befestigt ist und, wenn nöthig, künstliche Zähne trägt, allein der eigentliche Obturator (das zwischen vernähtes Gaumensegel und Pharynx eingeschobene Verschlussstück) ist nicht hohl, sondern besteht aus einem Stück weichen Velakautschuk, das in seiner Mitte einen Kern von Korkholz birgt. Zahnarzt Brugger ist der Ansicht, dass ein dermaassen beschaffener Obturator den Gaumenmuskeln und 
dem Constrictor pharyngis eine bessere und richtigere Anlehnung gebe, das Muskelspiel des Gaumensegels in wirksamerer Weise unterstiutze und so die verschiedenen Abstufungen der Klappengestalt besser zum Ausdruck kommen lasse, als ein hohler, allzu nachgiebiger Obturator. Er beansprucht sodann für seinen Obturator die Möglichkeit eines absolut dichten Abschlusses zwischen Mund- und Nasenhöhle, während gerade diese Leistung beim hohlen Obturator zu wünschen übrig lasse, da dieser von Zeit zu Zeit wieder aufgeblasen werden müsse und so die Muskeln in ihrem Anpassungsvermögen nicht zur Ruhe kommen lasse. Auch sind beim hohlen Obturator nicht alle Partieen der Oberfläche gleich elastisch; er wird daher bei energischen Verschlüssen, besonders im luftleerem Zustand, leicht Faltungen bekommen. So entstehende Rinnen können nicht immer durch die vorrückenden Muskeln ausgefüllt werden und es muss als nothwendige Folge hernach Luft durch die Nase abfliessen. Mit dem Schiltsky'schen Obturator hat der Brugger'sche Obturator die grosse Leichtigkeit und weiche Consistenz gemeinsam, er biegt sich so bequem und so reizlos wie dieser, dabei ist er leichter zu reinigen, dauerhafter und muss nicht von Zeit zu Zeit geöffnet und aufgeblasen werden. ${ }^{1}$ )

So viel ist sicher, dass meine operirten und mit dem Bruggerschen Obturator versehenen Kranken mit wenig Ausnahmen schon nach kurzem Sprechunterricht ganz ohne Nasenton sprechen lernten.

Von grösster Wichtigkeit ist nun aber die Beobachtung, dass der Gaumenobturator nach längerem Tragen nicht etwa die Gaumenmusculatur ungünstig beeinflusst und schwächt, sondern im Gegentheil durch eine Art permanenter Massage, die er auf den Gaumen ausïbt, denselben kräftigt und leistungsfähiger macht, so dass nach einiger Zeit ein Abschluss der Nasenhöhle gegen die Mundhöhle auch ohne Obturator erfolgen und derselbe bleibend beseitigt werden kann. Und das ist es, was in jedem Falle erstrebt werden muss. Nicht genug zu verurtheilen sind daher auch dieVersuchegewisser Zahnärte, die Operation als überflüssig zu discreditiren und die Prothese an Stelle der Gaumenplastik zu stellen. Die Prothese kann und wird immer nur ein Nothbehelf sein!

Ich lasse hier noch kurze Krankengeschichten der operirten Kinder bis zu 14 Jabren folgen, während ich auf die Mittheilung der Operations- und Krankengeschichten der Erwachsenen, die geringeres Interesse bieten, verzichte.

1) Der Brugger'sche Obturator ist in neuester Zeit durch Zahnarzt W ella u er noch verbessert worden. S. Schweiz. Vierteljahrsschr. f. Zahnheilk. 7 . Bd. 1897. 


\section{Krankengeschich ten.}

\section{Kinder bis zu 2 Jahren.}

1. St., August, war zuerst Juni 1866, damals 4 Wochen alt, im Spital mit linksseitiger durehgeliender Unilateralspalte des harten Gaumens, medianer Spaltung des Velum und Spaltung der Oberlippe. Es wurde auf den Wunseh der Eltern die Ilasenscharte operirt und das Kind dann wieder nach Hause entlassen. Im 11. Lebensmonat wurde es zum zweiten Mal bei gutem Kräftezustand in's Spital aufgenommen. Juni 1867 Uranostaphylorrhaphie. Das Resultat war die vollständige Vereinigung des harten Gaumens, während die Naht des Velum wieder auseinander ging. Im Jahre 1871 sah ich den kleinen Patienten wieder. Es wird neuerdings die Herstellung des harten Gaumens festgestellt und die Annäherung der wieder auseinander gegangenen Velumränder. Leider können sich die Eltern zu einer zweiten Operation nicht verstehen. Die Sprache ist verständlich, aber stark näselnd.

2. G., Ernst, mit Lippenspalte und durchgehender linksseitiger Unilateralspalte des larten Gaumens und medianer Spaltung des weichen Gaumens geboren, wurde kurz nach der Geburt an der Hasenscharte operirt. November 1866, 23 Monate alt, kam er in's Spital. Ich wollte bei dem anämischen und schlecht genährten Kind zuerst den harten Gaumen herstellen und erst später die Staphylorrhaphie folgen lassen und so wurde denn am 29. November die Uranoplastik gemacht und gelang vollständig. Bevor an eine zweite Operation gedacht werden konnte, nahmen die Eltern das Kind nach Hause und erst als 21 jähriger Gärtner kam Patient neuerdings in meine Behandlung. Die beiden Hälften des weichen Gaumens standen $1 / 2 \mathrm{~cm}$ auseinander. Am 13. Januar 1886 Staphylorrhaphie, die vollständig gelingt, und am 25. Januar Excision der hässlichen, strahligen Hasenschartennarbe. Nachher verlor ich den Kranken aus den Augen und erfuhr erst später, dass er, ohne Sprachunterricht zu nehmen, eine Zeitlang einen Gaumenobturator trug, den er aber bald wieder ablegte, da er ihm beim Essen und Trinken sehr hinderlich war und beim Arbeiten den Athem beengte. Die Sprache soll noch näselnd, aber gut verständlich sein, am besten spreche er im Affect.

3. H., Anton, 17 Monate alt, mit einer reclitsseitigen, durchgehenden Gaumenspalte und Hasenscharte auf die Welt gekommen, wurde 10 Tage nach der Geburt von der Hasenscharte befreit. Im 17. Lebensmonat Naht der Gaumenspalte, die nur die Vereinigung des harten Gaumens erzielte. 3 Jahre später starb Patient an Diphtherie.

4. F., Therese, wurde mit einer Bilateralspalte des harten Gaumens mit Spaltung des Gaumensegels in der Mittellinie und mit doppelseitiger Spaltung der Oberlippe geboren. Operation der Hasenscharte 9 Wochen nach der Geburt am 12. Januar 1877 mit leidlichem Erfolg. Im Alter von 23 Monaten Uranostaphylorrhaphie am hängenden Kopf. Misserfolg, die ganze Nalitlinie geht wieder auseinander. Ich sah die Kranke nie wieder. Sie soll im Jahre 1891 nach Amerika ausgewandert und seit einigen Jahren verheirathet sein. Die Sprache war und blieb undeutlich und näselnd.

5. G., Elise, 3 Monate altes Kind, das mit Kuhmilch, Fenchelthee und Brei aufgefüttert worden war. Der weiche und der harte Gaumen, mit Ausnahme des Alveolarfortsatzes, gespalten. Breite der Spalte fast durehweg $1^{1 / 2} \mathrm{~cm}$. Am 19. März 1878 Uranostaphylorrhaphie. Die Naht geht aus- 
einander. Vollständiger Misserfolg. Das Kind stirbt $1 / 4$ Jahr später zu Hause an catarrhalischer Pneumonie.

6. Schr., Gebhard. Durchgehende linksseitige Spalte des harten Gaumens mit Spaltung des Gaumensegels und der Lippe. 8 'Tage nach der Geburt Operation der Hasenscharte, die vollständig gelingt. $7 \frac{1}{2}$ Monate später Uranostaphylorhaphe am hängenden Kopf mit Hilfe des Whiteheadschen Speculums. Die Spalte war auf der Höhe der Zahnreihe $4 \mathrm{~mm}$, in der Mitte des harten Gaumens $12 \mathrm{~mm}$, am Uebergang des harten in den Gaumen $14 \mathrm{~mm}$, in der Mitte des weichen Gaumens $12 \mathrm{~mm}$ breit. Das Resultat war eine vollständige Vereinigung des lıarten Gaumens und eine Annäherung der Velumränder, die in der Mitte noch $7 \mathrm{~mm}$ und an der Uvula noch $8 \mathrm{~mm}$ auseinander standen. Der Knabe kam erst in 5. Jalıre zum Gehen, machte mit 51/2 Jahren eine Lungenentzïndung durch und besuchte vom 7. Jahre an die Schule, in der er ordentlich mitkam. Mit 12 Jahren sah ich ilın wieder. Die Sprache ist verständlich, aber stark nasal. Es besteht noch eine spitzwinkelige Spalte des weichen Gaumens, deren grösste Breite an den Zäpfelienhälften ea. $1 \mathrm{~cm}$ betrïgt. Bei Intoniren von $A$ lıeben sich beirle Zäpfchenhı̈lften kräiftig. Der Vater des Knaben geht auf den Vorschlag, den weichen Gaumen nochmals zu nähen, nicht ein.

7. F., Otto, 11 Tage alt, in's Spital aufgenommen am 3. April 1891. Vor 11 'Tagen geboren mit doppelseitjger Hasenscharte, von denen die rechte die Hälfte, die linke $2 / 3$ der Oberlippe einnimmt und mit Spaltung des weichen Gaumens und des harten Gaumens bis zur Mitte des 1'rocessus palatini. Die Breite der Spalte beträgt an der Grenze des harten und weichen Gaumens $7 \mathrm{~mm}$, in der Mitte des weichen Gaumens $9 \mathrm{~mm}$, an den Zäpfelienhälften $9 \mathrm{~mm}$.

Am 9. April 1891, also am 17. Lebenstage, Uranostaphylorrhaphie in Chloroformnarkose am hängenden Kopf. Die Vereinigung gelingt in dieser einen Sitzung. Am 4. Mai 1891 Operation der Hasensehrute, die ein sehr zufriedenstellendes Resultat ergiebt. Entlassung am 30. Mai.

Am 13. Mai 1901 sah ich den Knaben wierler. El ist für sein Alter klein und mager, sieht aber gesund aus. $\mathrm{Er}$ geht schon 5 Jahre in die Schule, ist aber ein schlechter Schüler und schon mehrmals sitzen geblieben, was zum Theil mit seiner: Schwerhörigkeit zusammenhängen magr. Er hört eine stark schlagende Ankeruhr rechts auf $10 \mathrm{~cm}$ Distanz, links nur beim Andrücken an's Ohr. Kopf und Kiefer wohlgebildet. Die Zähne des Oberkiefers prominiren in normaler Weise vor denen des Unterkiefers. Ein Blick in den Mund lässt einen gut gebildeten, etwas steilen Oberkiefer und ein breites Velum mit deutlich ausgesprochenen Gaumenbögen erkennen. Bein Intoniren zieht sich das Velum stark nach oben und hinten. Die Sprache hat einen stark nasalen Beiklang, ist aber nicht gerade unangenelım und mühsam anzuhören Der Sprachlehrer stellt ohne Zuhilfenahme eines Gaumenobturators durch systematischen Sprechunterricht allein eine normale Sprache in sichere Aussicht, aber die einfältigen Eltern können sich nicht dazu verstehen.

Nr. 8 siehe oben.

9. 'T., Anna, 22 Monate alt, in's Spital aufgenommen den 3. April 1895. Sonst gesund und von blülıendem Aussehen, leidet an einer Spalte des weichen Gaumens und der horizontalen Platte des Gaumenbeins. Die Länge der Fistel beträgt $3 \mathrm{~cm}$, die Breite ganz vorn, spitz zulaufend, $3 \mathrm{~mm}$, Mitte 5 , hinten $6 \mathrm{~mm}$. Das Kind kann nur mit Flasche trinken, mit dem Löffel 
eingeführte Flüssigkeit kommt grösstentheils wieder zur Nase heraus. Die wenigen Worte, die das Kind stammelt, sind fast unverständlich.

Operation in Chloroformnarkose, am hängenden Kopf, mit Benützung des $W$ hitehead am 8. April 1895. 8 Nähte. Entfernung derselben am 6.-7. Tage. Completer Schluss der Spalte. Am 8. Mai 1902 schreibt der Vater von dem Kinde: "Es spricht allerdings noch nicht gut, aber immerhin so, dass sie es in der Schule verstehen. Es kommt noch vor, dass es ausgelacht wird, aber in der Schule nie. Bezüglich des Kiefers und der Zähne ist alles in Ordnung." Juli 1902 sah ich das Kind selbst. Es besteht ein hohes, activ sehr bewegliches Velum mit geschlossenem harten Gaumen. Die Sprache ist undeutlich und stark näselnd. Die Zälne des Oberkiefers überragen die Unterkieferzahnreihe.

10. Z., Carl, 6 Monate alt, aufgenommen den 31. October 1888. Kam mit durchgehender linksseitiger Gaumenspalte und linksseitiger Hasenscharte zur Welt. In der Familie des Kindes sind keine derartigen oder anderweitigen Missbildungen beobachtet worden. Trotz Hasenscharte und Gaumendefect soll die Nahrungsaufnahme keine erhebliehen Schwierigkeiten gemacht haben und das Kind sieht ordentlieh genährt aus. Die Breite der Gaumenspalte beträgt am Process. alveolaris $4 \mathrm{~mm}$, in der Mitte des harten Gaumens $10,5 \mathrm{~mm}$ und an der Grenze des harten und weichen Gaumens $11 \mathrm{~mm}$.

Am 1. November Operation der Hasenscharte, die ein sehr gutes Resultat giebt, am 1. December Uranostaphylorrhaphie am hängenden Kopf in Chloroformnarkose. Auch diese Vereinigung gelingt bis auf ein kleinerbsengrosses Loch an der Grenze des liarten und weichen Gaumens, das sich in 8 Tagen auf Stecknadekopfgrösse geschlossen hatte. Nach 14 Tagen wird das Kind mit geschlossenem Gaumen nach Hause entlassen. 8 Tage später bildeten sich am Koplf und Nacken 6 Furunkel und von diesen ausgehend ein Erysipel, dem das Kind nach weiteren 4 Tagen erliegt.

11. Sch., Max, 3 Monate alt. Gutgenährtes Kind mit durchgehender rechtsseitiger Gaumenspalte und doppelter Hasenscharte. Operation der Gaumenspalte am 26. Juni 1890, und zwar Anfrischung der Ränder ohne Narkose in aufrechter Stellung, dann Narkose und Loslösung der Lappen am hängenden Kopf. 7 Nähte. Die Vereinigung gelingt bis auf ein $4 \mathrm{~mm}$ langes und $3 \mathrm{~mm}$ breites Loch an der Grenze des harten und weichen Gaumens. Dann Operation der Hasenscharte am 17. Juli 1890. Heilung mit selr gutem, kosmetischem Resultat. Am 9. August 1890 Sehluss des Loches an der Grenze des harten und weichen Gaumens. Entlassung, völlig geheilt, am 22. August 1890. Stirbt April 1891 zu Hause an Meningitis.

12. M., Jakob, 9 Monate alt, hat eine durchgehende linksseitige Gaumenspalte und hatte eine linksseitige Hasenscharte, die 14 Tage nach der Geburt operirt worden war. Die steil gestellten Process. palatini der Oberkieferhälften sind sehr kümmerlich und die Spalte ist stellenweise fast so breit, wie die Process. palatini zusammen.

Operation am hängenden Kopf in Chloroformnarkose. Es werden im Ganzen 10 Nähte angelegt, eine auf der Hinterseite der Uvula. Entfernung derselben nach 7 Tagen. Bis auf eine kleine Spalte dicht hinter der Lippe, die wegen Schonung der Arter. nasopalatinae bleiben musste, gelingt die Vereinigung und das Kind wird nach Hause entlassen. 
6 Wochen später erliegt es laut ärztlichem Bericht einer Lungenentzündung.

\section{Kinder von 2-6 Jahren.}

1. W., Therese, $3 \frac{1}{2}$ Jahre alt, aufgenommen den 17. März 1881. Durehschnittlich $1-2 \mathrm{~cm}$ breite, $2 \mathrm{~cm}$ hohe Spalte des weiehen Gaumens. Patientin verschluckt sich oft und ein Theil der eingegebenen Flüssigkeit fliesst durch die Nase ab. Näselnde, fast unverständliche Sprache.

Staphylorthaphie den 2. April 1881 am hängenden Kopf in Chloroformnarkose. In der Nacht vom 5./6. ein heftiger Hustenanfall mit Durchreissen der Nähte. Entlassung am 13. April.

2. K., Peter, 6 Jahre alt, aufgenommen den 27 . Februar 1888 . Kräftiger Knabe mit angeborener Spaltung des Velum. Der harte Gaumen normal, Zahnstellung desgleichen. Die Sprache ausserordentlich undeutlich und stark nasal, die Vocale alle nasal, $\mathrm{i}$ fast unverständlich, sämmtliche Mediae b, d, q und Tenues $\mathrm{p}, \mathrm{t}, \mathrm{k}$ mit starkem Zischen der Luft durch die Nase. Am 6. März Staphylorrhaphie am hängenden Kopf in Chloroformnarkose. Entfernung der Nälte am 13. März. Schöne Vereinigung der ganzen Spalte unit wohlgebildeter langer Uvula. Geringe Sprachverbesserung in den ersten der Operation folgenden Wochen. Sprechunterricht wird refusirt. Ueber das weitere Schicksal des Patienten schreibt Dr. Lardelli: K. ist Schmied geworden, seine Sprache soll, wie ich von verschiedenen Seiten hörte, eine ganz ordentliche sein, namentlich wenn inan bedenkt, dass K. geistig beschränkt ist.

3. W., Max, 3 1/2 Jalne alt, aufgenommen den 4. Februar 1889, wurde geboren mit einer durchgehenden, linksseitigen Gaumenspalte und linksseitiger Hasenscharte. Im Alter von 3 Monaten wirde vom Hausarzt die Hasenseharte operirt mit gutem Erfolg.

Es handelt sich un einen schlecht genährten, schwächlichen Knaben mit starkem Foetor oris, eitrigem Ohrenfluss und ehronisehem Nasen- und Rachencatarrh. Die durchgehende Gaumenspalte ist am Process. alveolaris $6 \mathrm{~mm}$, in der Mitte des harten Gaumens $11 \mathrm{~mm}$ und an der Grenze des harten und weichen Gaumens $14 \mathrm{~mm}$ breit. Patient giebt nur unarticulirte Isaute von sich.

Es werden zunäehst 10 Tage lang Salicylspülungen des Mundes und der Nase am hängenden Kopf vorgenommen. Dann am 15. Februar erster Act der zweizeiligen Uranostaphylorrhaphie am hängenden Kopf in Chloroformnarkose, Loslösung und Annäherung der Lappen, Fixation in dieser Stellung durch 2 Nähte ohne Anfrischung. 8 Tage später zweiter Act: Anfrischung und nochmalige Lösung der Lappen, Anlegung der Naht. Am Abend des Operationstages Temperatur $39,5^{\circ}$, am folgenden Morgen 38,40, Abends $38^{\circ}$, dann fieberfrei. Am 5. Tage zeigen sich in der Nahtlinie 2 Löcher, doch finden sich 2 feste Brücken, eine an der Uvula und eine vorn. Am 7. Tage reissen auch diese Brücken ein, so dass der Misserfolg vollständig ist. Die Spalte ist aber enger, hat in der Mitte des harten Gaumens eine Breite von 7, am Uebergang des harten in den weichen Gaumen eine Breite von $9 \mathrm{~mm}$. Neulich sah ich den Kranken, der sich zu einer zweiten Operation nicht verstehen konnte. Die Sprache ist stark nüseind, unangenehm zischend und bei raschem Lesen fast unverständlich. 
4. L., Christine, $31 / 2$ Jalre alt, aufgenommen den 17. November 1890 , stammt aus gesunder Familie, in der bis jetzt keine Missbildungen vorkamen, das Kind selbst war bis jetzt nie ernstlich krank und sieht gesund und kräftig aus. Es besteht ohne Hasenscharte eine doppelseitige Spaltung des harten Gaumens, mit Ausnahme des Alveolarfortsatzes. Der Vomer, der in der Tiefe der breiten Gaumenspalte als feine rothe Leiste sichtbar ist, theilt den Spalt in 2 Hälften. Die ziemlich schmalen Process. palatini tler Oberkiefer sind steil aufwärts gerichtet. Breite der Spalte zwischen den Zäpfehenhälften $15 \mathrm{~mm}$, an der Grenze des weichen und harten Gaumens $16 \mathrm{~mm}$, nahe dem vorderen Ende der Spalte $7 \mathrm{~mm}$. Sprache stark näselnd und beinahe unverständlich.

Am 26. November 1890 Uranostaphylorrhaphie in einer Sitzung. Anfrisehung, Loslösung der Lappen, 9 Seidenknopfnähte und 2 an der Hinterseite der Uvula mit krummen Nadeln und Nadelhalter in Chloroformnarkose an hängenden Kopf. 'Am 6. Tag Entfernung der Jodoformmèchen, am 7. der Nähte. Tarlelloser Schluss der ganzen Spalte.

Am 17. November 1891 zeigt sich das Kind wieder. Der Gaumensegel macht beim Intoniren kräftige Bewegungen, die Sprache verständlich, aber mit starkem Nasenton.

Am 17. Mai 1901 stellt sich das nun 15 jährige Mädehen wieder vor. Es sieht gesund und kräftig aus, besuchte die Volksschule und später die Repetirschule. Sie besitzt einen ziemlich steilen, harten Gaumen und ein wohlgebildetes Velum mit scharf gezeichneten Gaumenbogen. Das Velum hat eine Höhe von $21 / 2 \mathrm{~cm}$ und zeigt beim Intoniren kräftige Bewegungen und es ist ein sehr stark entwickelter Pharynxwulst vorhanden. Hört Flüsterstimme auf Zimmerdistanz und eine schwach schlagende Ankeruhr beiderseits auf $34 \mathrm{em}$ Distanz. Sie liest ohne Verziehung der Gesichtsmuskeln mit klangvoller Stimme geläufig und gut verständlich, doch näselt sie noch stark und b und t spricht sie mit zischendem Nasendurchschlag, der Spiegel beschlägt sich bei allen Vocalen und das Zungen-r fehlt. Keine Atrophie des Oberkiefers und beim Biss stehen die Zahnreihen genan aufeinander.

Auf meinen Wunsch nahm das Mädchen einen 14 tägigen Sprechunterricht bei Herrn Schittenmüller mit glänzendem Resultat. Das Zungen-r war nach dem Unterricht nomal und alle übrigen Buchstaben rein, der zischende Nasendurchsellag versehwunden.

5. N., Bertha, 6 Jahre alt, aufgenommen den 1. Juni 1896, leidet an einer angeborenen Gaumenspalte, die das Velum trennt und bis zur Mitte der Process. palatini reicht. Das Mädchen ist zart und schlecht genährt.

Die Spalte ist im vorderen Drittheil $14 \mathrm{~mm}$, im hinteren Drittheil $25 \mathrm{~mm}$ breit. Patientin spricht fast unverständlich und stark nasal mit schnaubenden Nasengeräuschen.

Am 3. Juni Uranostaphylorrhaphie in Chloroformnarkose am hängenden Kopf mit Loslösung der mucös periostalen Lappen, Anfrischung und Naht. 8 Nähte, 2 an der Rückseite der Uvula. Die Nähte werden am 8. Tage entfernt. Vereinigung der Spalte bis auf ein $10 \mathrm{~mm}$ langes und $3 \mathrm{~mm}$ breites Loch am harten Gaumen. Patientin sollte in 6 Wochen wieder zur Nachoperation kommen, kam aber nicht. Später hörte ich, dass das noch vorhandene Loch zu Hause operativ geschlossen wurde, lass aber keine 
wesentliche Besserung der Sprache bemerkbar sei. Sprechunterricht hat das Mädchen nie gehabt.

6. I., Hans, $53 / 4$ Jahre alt, aufgenommen den 2. Mai 1897. Gut entwickelter, kräftiger Knabe. Durchgehende linksseitige Gaumenspalte, die vorn zwischen Eckzahn und Schneidezahn beginnt und hier nur $2-3 \mathrm{~mm}$

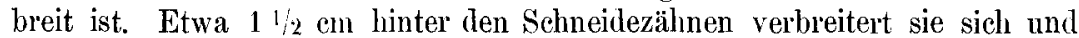
ist $3 \mathrm{~cm}$ hinter den Schmeidezähnen 11 muı breit, an der Grenze zwischen hartem und weichem Gaumen $14 \mathrm{~mm}$. Die Zäpfehenhälften stehen $9 \mathrm{~mm}$ von einander ab. Sprache fast unverständlich, nasal, sämmtliche Vocale stark nasal, von den Consonanten nur $m$ und $\mathbf{n}$ rein.

Am 5. Mai 1892 Uranostaphylorrhaphie am hängenden Kopf in Narkose. 7 Nähte. Am 6., 7. und 8. Tage Salicylspülungen. Am 7. und 8. Tage Entfernung der Nähte. Anı 13. wird der vollständige Schluss der Spalte festgestellt mit Ausnahme eines kleinen Spaltes im Alveolarfortsatz des Gaumens. Patient wird dem Sprachlehrer übergeben und nimmt 6 Wochen systematischen Sprechunterricht. Die Fortschritte im Sprechen sind aber sehr gering, weil Patient nach Angabe des Lehrers geistig noch sehr zurück ist. Nach Ablauf dieser 6 Wochen wird am 7. Juni 1897 diese kleine Spalte durch eine zweite Operation in einer Sitzung geschlossen und Patient mit vollständig vereinigter Gaumenspalte am 23. Juni 1897 nach Hause entlassen.

Am 17. Juni 1901 schreibt mir der Vater des nun 11 jährigen Knaben Folgendes: "Der Knabe hat im vorigen Jahre bei einer hiesigen Lehrerin wöchentlich " 1 Stunde Sprechunterricht genommen und relativ günstige Erfolge erzielt. Er wird im nächsten Frühjahr noch einen speciellen Curs bei Herrn Schittenm üller nehmen müssen, was wir eigentlich schon für dieses Jahr geplant hatten. Es wurde nie ein Obturator verwendet.

Der Knabe spricht jetzt verhältnissmässig recht gut, wenn er langsam spricht und sich Mühe giebt. Die Stimme ist klangvoll, ohne jeden näselnden Beiklang. Die Vocale sind ohne Ausnahme von tadellosem Klang; ebenso die meisten Consonanten, mit Ausnahme von g und $\mathrm{s}$. Er ist ein normaler, guter Schüler in allen Fächern, mit Ausnahme des Singens. Singen kann er nicht, weil er nieht sehr fein hört und zeitweise deshalb in lBehandlung stand. Körperlich hat sich der Knabe gut entwickelt und ist auch geistig nicht zurückgeblieben."

7. H., Josephine, 5 Jahre alt, aufgenommen den 1. September 1897. In der Ascendenz und bei den Geschwistern sind keine Missbildungen zur Wahrnehmung gekommen. Anfänglich machte die Ernährung des Kindes grosse Schwierigkeiten. Es besteht eine mediane Spalte, die in der Mitte des harten Gaumens beginnt und durch das ganze Gaumensegel geht. Die Länge der Spalte beträgt $6 \mathrm{~cm}$. Vorn ist sie $1 \mathrm{~cm}$, an der Grenze des harten und weichen Gaumens $1,5 \mathrm{~cm}$ breit und die Zäpfchenhälften stehen $1,9 \mathrm{~cm}$ auseinander. Sprache näselnd und unverständlich. Alle Vocale nasal. Von den Consonanten sind nur die Resonanten $m$ und $n$ rein. Beim Aufsagen eines Gebetes sind nur einzelne Worte verständlich. Geistig scheint das Kind sehr wenig vorgeschritten, es ist ausserordentlich schüchtern, vergrämt, weint viel.

Am 7. September 1897 Uranostaphylorhaphie in Chloroformnarkuse an hängenden Kopf. Entfernung der Nähte am 7. und 8. Tage. Die 
Vereinigung gelingt bis auf ein bohnengrosses Loch im harten Gaumen, das am 7. Januar 1898 in einer Sitzung geschlossen wird, so dass nun ein vollständiger Gaumen mit schön ausgeprägten Areus palatoglossi und normal langer Uvula hergestellt ist.

Nachdem im Jahre 1902 die Sprache des Kindes noch nasal und schwer verständlich ist, obwohl sie die Vocale a, 0 und e, wie auch die Consonanten b, d, p einzeln schon absolut rein bildet, wird ein $\mathbf{5}$ wöchentlicher Sprechunterricht bei Herrn Schittenmüller genommen. Das Resultat ist eine absolut reine Sprache, die auch sehon ziemlich fliessend ist und zwar sowohl in ilırem Dialect (Schafflausen) ebenso gut, wie im Schriftdeutschen. Auch singt sie sehr hübsch. Der Gaumen wurde während des Sprechunterrichts kräftig massirt.

S. B., Ruppert, $41 / 2$ Jahre alt, aufgenommen den 19. December 1898 , kam mit einer durchgehenden linksseitigen Gaumenspalte und linksseitiger Hasenscharte zur Welt. Die Hasenscharte wurde 4 Wochen nach der Geburt mit sehr gutem Resultat operirt.

Kräftig entwickelter, aufgeweckter Knabe mit vollständiger Spaltung des harten und weichen Gaumens. Im Process. alveolaris ist die Spalte nur $3 \mathrm{~mm}$ breit, $2 \mathrm{~cm}$ von der Zahnreihe entfernt $13 \mathrm{~mm}$ und an der Grenze des harten und weichen Gaumens $20 \mathrm{~mm}$. Sprache sehwerfällig, stark näselnd und schwer verständlich. Sämmtliche Vocale nasal, die Zungenweichgaumenconsonanten unrein oder können nicht gebildet werden.

Am 26. December 1898 Uranostaphylorrhaphie am hängenden Kopf in Chloroformnarkose mit 6 Nälten und 2 Nähten an der Hinterseite der Uvula. Ganz vorn im Process. alveolaris bleibt, wie bei jeder durehgehenden Spalte aus Rüeksicht der Ernährung der beiden Lappen, ein kleiner Schlitz, zll dessen glücklicher Beseitigung l'atient sieh nochmals am 5. October 1900 in's Spital aufnehmen liess. Sein Hausarzt, Herr Dr. EImi ger, schrieb mir Juni 1901 über diesen Knaben Folgendes: Der Knabe hat nie einen Obturator getragen und auch keinen systematischen Sprechunterricht genossen. Seine Sprache ist ziemlich deutlich und hat nasalen Beiklang. Die Buchstaben $\mathrm{g}, \mathrm{k}$ und $\mathrm{r}$ sind ziemlich rein, weniger gut $\mathrm{d}, \mathrm{t}$ und $\mathrm{h}$. Ich habe einige Sprechübungen mit dem Kleinen gemacht und eingesehen, dass mit wenig Mühe ein vorzügliches Resultat zu erzielen wäre. Das Gaumensegel macht beim Intoniren kräftige Bewegungen.

9. D., Walther, 3 Jahre alt, aus gesunder Familie stammend, in der Missbildungen gänzlich unbekannt sind, von 6 Geschwistern das viertälteste Kind.

Bei der Aufnahme am 19. September 1901 zeigt sich, dass der ganze weiche Gaumen und die horizontale Platte des Gaumenbeins median gespalten sind. Die grösste Breite der Spalte, etwa in der Mitte, beträgt $16 \mathrm{~mm}$. Patient spricht mit Verziehen der Gesichtszüge stark näselnd und undeutlich. Sämmtliche Vocale haben starken Nasenbeiklang, am wenigsten 0 und $\mathrm{u}$. Die Consonanten $\mathrm{b}, \mathrm{d}, \mathrm{g}, \mathrm{r}$ kann er nicht bilden, $\mathrm{p}, \mathrm{t}, \mathrm{k}$ haben zischenden Nasendurchschlag und sind von starkem Verziehen der Oberlippe begleitet u. s. w.

Uranostaphylorrhaphie am 21. September 1901 am hängenden Kopf in Chloroformnarkose mit 5 Nähten und 2 an der Hinterseite der Uvula. Vollständiger Sehluss der Spalte his auf ein steeknadelkopfgrosses Loch 
im harten Gaumen, das 1/2 Jahır später ohne Schwierigkeit zum Schluss gebraclit wurde. Soll mit 5 Jahren Sprechunterricht bekommen. Das Endresultat ist ein wohlgebildeter Gaumen mit schön geschwungenen Arc. palato glossi und palato pharyng. und wohlgebildeter Uvula.

10. A., Peter, 4 Jahre alt, aufgenommen den 29. October 1901. Eine Cousine des Knaben besitzt ebenfalls eine Gaumenspalte, sonst keine Missbildungen in der Familie. Der Knabe war immer zart, aber sonst gesund.

Spaltung des weichen Gaumens und des harten Gaumens bis zur Mitte der Process. palatini. Breite der Spalte an der Grenze des harten und weichen Gaumens $18 \mathrm{~mm}$, hinten ist die Spalte bei ruhiger Haltung der Velumhälften noch $1 / 2 \mathrm{em}$ breiter. Der Knabe verschluckt sich mitunter beim Essen, auch kommt zuweilen Milch zur Nase heraus. Sprache sehr undeutlich und näselnd. Mit Ausnahme von $\mathrm{l}, \mathrm{m}$ und $\mathrm{u}$ sind alle Buchstaben mehr weniger unrein.

Uranostaphylorrhaphie am 8. November 1901 in Chloroformsauerstoffnarkose am hängenden Kopf. 9 Nähte, davon 2 an der Hinterseite der Uvula. Nachdem am 11. November bei schönem Schluss der Spalte die letzten Nähte in Narkose entfernt worden waren, sprengt der widerspenstige und ungeberdige Knabe dureh lautes Brüllen die Naht der Uvula in der unteren Hälfte des weichen Gaumens. Nachoperation mit günstigem Erfolg Mai 1902. Soll später Sprechunterricht bei Herrn Schittenmüller bekommen.

11. D., Heinrich, 4//2 Jahre alt, aufgenommen den 6. Februar 1902. Sonst gesundes Kind gesunder Eltern. Spalte des weichen Gaumens und der horizontalen Platte des Gaumenbeins. Die beiden Velumbälften etwas verkümmert, Breite der Spalte in der Mitte $19 \mathrm{~mm}$. Abstand des weichen Gaumens von der hinteren Pharynxwand $2 \mathrm{~cm}$. Sehr gut entwickelter Pharynxwulst.

Sprache stark näselnd, fast unverständlich, mühsam anzuhören und von Gesichtsgrimassen begleitet. Alle Vocale nasal, b, p, f, s, und $\mathrm{z}$ mit zischendem Nasendurchschlag.

Am 11. Februar 1902 Uranostaplıylorrhaphie am hängenden Kopf in Chloroformnarkose. Einige Minuten nach Beginn der Narkose Asphyxie dureh Herabdrücken der Zunge dureh den Zungenfortsatz des Whitehead. 7 Nähte, 1 an der Hinterseite der Uvula. Am 8. Tage wird eine kleine bohnengrosse Gangrän des rechten Wundrandes an der Grenze des harten und weichen Gaumens bemerkt, die nach Abstossung ein kleines Loch zurïcklässt, das sich später zu Hause von selbst schliesst. Sonst schöne Vereinigung des ganzen Gaumens incl. Uvula mit schön gezeichnetem Gaumenbogen. Das Velum hat jetzt eine Höhe von $2^{1 / 2} \mathrm{~cm}$. Hatte bis jetzt noch keinen Sprechunterricht, soll aber später solchen bekommen.

\section{Kinder von 7-14 Jahren.}

1. B., Ludwig, 12 Jahre alt. Kräftig entwiekelter, intelligenter Knabe. Angeborene Spaltung des weichen Gaumens und der horizontalen Platte des Gaumenbeins.

Uranostaphylorrhaphie am 21. November 1866. Die Vereinigung der ganzen Spalte erfolgt anstandslos. 
Es war mir nicht möglich, über das weitere Schicksal dieses Operirten Erkundigungen einzuziehen.

2. Huber, Johannes, 11 Jahre, von Altersweilen. Linksseitige, durchgelıende Spalte des harten Gaumens mit Spaltung des Velum.

Uranostaphylorrhaphie am 27. October 1868. Der Schluss der Spalte wird durch 2 Operationen erzielt. Die momentane Wirkung auf die Sprache war fast Null. Er lebt noch im Jahre 1902 und ist Hausirer. Er spricht deutlich, aber nasal.

3. S., Bertha, 61/2 Jahre alt, gesundes Kind. Spaltung des weichen Gaumens.

Uranostaphylorrhaphie am 10. April 1876. Vereinigung bis auf eine erbsengrosse Oeffnung im oberen Wundwinkel, die im August 1876 operativ geschlossen wird. Ein Bericht des Mannes der indessen verheiratheten Patientin vom Juni 1891 besagt, dass ohne Obturator und Sprechunterrieht die Sprache geläufig und deutlich, aber etwas näselnd sei. Die Frau hat indessen 2 gesunde Kinder geboren.

4. E., Ida, 12 Jahre alt, gesundes Kind gesunder Eltern, aufgenommen den 1. August 1582, kam mit Hasenscharte und Spaltung des weichen Gaumens und der horizontalen Platte des Gaumenbeins zur Welt. Die Hasenseharte wurde mit bestem Erfolg im 1. Lebensjahlre operirt. Uranostaphylorrhaphie am 21. August 1882 in Chloroformnarkose am hängenden Kopf, 5 Nähte. Die Vereinigung gelingt auf's Schönste. Die Sprache blieb aber näselnd und unverständlich, wie vor der Operation; alle Vocale nasal, sehr viele Consonanten mit Nasendurehschlag. September 1882 wurde dann ein B rugger'scher Obturator angefertigt und Patientin erhielt einen 4 wöchentlichen Sprechunterricht bei Herrn Wang. Sie wurde mit völlig normaler Sprache aus diesem Unterricht entlassen.

5. K., Josephine, 8 Jalıre alt, aufgenommen den 27. Juni 1885 , für ihr Alter etwas klein und schwächlich, leidet an einer angeborenen Gaumenspalte, die den ganzen weichen Gaumen und die horizontale l'latte des Gaumenbeins betrifft. Die Sprache ist sehwer verständlich und stark nasal.

Uranostaphylorrhaphie in aufrechter Stellung mit Cocainisirung der Schleimhaut. Die Vereinigung erfolgt bis auf ein kleines Loch, an der Grenze des harten und weichen Gaumens, das sich von selber schliesst und durch eine beschränkte Gangrän des rechten mucös periostalen Lappens entstanden war.

Am 25. Juni 1901 erhielt ich durch Herrn Dr. Walder folgenden Bericht über die Operirte: „Aus dem 8jährigen Mädehen vom Jahre 1885 ist eine gesunde, 25 jährige Jungfrau des Jahres 1901 geworden, welche erklärt, dass sie, ohne Sprechnnterricht genossen zu haben und ohne $\mathrm{Ob}$ turator, für Jedermann verständlich spreche. Ich kann das Letztere bestätigen. In unserer ganzen Conversation war nur das Wort 'Vergolderei' sehwer' verständlich. Dagegen ist das 'Timbre der Sprache stark näselnd, und zwar sowohl für Vocale wie Consonanten, welche aber deutlich ausgesprochen werden, auch $\mathrm{g}, \mathrm{k}, \mathrm{d}, \mathrm{t}, \mathrm{s}$, sowohl für sich allein, als in den Worten: guten Tag, danke, Gesang, nur $\mathrm{r}$ ist mangelhaft. Der weiche Gaumen ist wohlgebildet und bewegt sich kräftig."

6. H., Ottilie, 8 Jahre alt, aufgenommen den 16 . Februar 1886, leidet an angeborenerLues, sich jetzt noch durch schmerzhafte Knochenauftreibungen 
an den beiden Tibiae und dem rechten Radius kennzeichnend und an einer angeborenen Gaumenspalte, die den weichen Gaumen und die horizontale Platte des Gaumenbeins betrifft.

Uranostaphylorrhaphie am 19. April 1886. Vollständiger Sehluss der Spalte. October 1902 stellt sich Patientin wieder vor. Sie arbeitet gegenwärtig in einer Seidenfabrik und hat weder einen Obturator sich anfertigen lassen, noch Sprechunterricht genommen. Sie besitzt ein wohlgebildetes Gaumensegel von etwas über $3 \mathrm{~cm}$ Höhe, das bei ruliger Lage $1,5 \mathrm{~cm}$ von der hinteren Pharynxwand absteht. Beim Intoniren von a macht das Gaumensegel kräftige Bewegungen nach hinten und oben. Stark ausgesprochener Pharynxwulst.

Die Sprache ist fliessend und deutlich, aber stark näselnd, auch verzieht sie beim Sprechen den Mund und faltet die Stirne. $s, t$, d sind von zischendem Nasendurchschlag begleitet.

7. B., Victor, 11 Jahre alt, aufgenommen den 17. November 1887. Die Grosseltern sind gesund, desgleichen die Eltern. Von den 18 Geschwistern des Vaters hat keines einen Bildungsfehler. Die 3 Geschwister des Patienten sind ebenfalls gesund. Die Mutter glitschte, während sie mit dem Knaben schwanger ging, aus und fiel zu Boden, ein sogenanntes Versehen kam nicht vor. Die Geburt ging gut und ohne ärztliehe Hilfe von Statten. Der Bildungsfehler wurde bei dem Patienten erst nach 1/2 Jahr bemerkt. Der Vater ging dann mit ihm zu einem Chirurgen, der erklärte, es sei nichts zu machen. Mit $5 / 4$ Jahren lernte der Knabe sprechen, aber sehr mangelhaft, er war schwer zu verstehen. Mit 6 Jahren besuchte er die Sehule, allein der Lehrer verstand ihn oft nicht, von den Mitschülern wurde er seiner mangelnden Sprache wegen oft ausgelacht. Er fühlte sich deshalb gekränkt, wurde oft heftig, weinte viel, dabei war er befähigt, kam gut nach und galt als tüchtiger Schüler. Mit 10 Jahren wurde das Sprechen noch schlechter, im höchsten Grade undeutlich, von dumpfem hohlen Klang, so dass nur Leute, die beständig in seiner Nähe waren, ihn überhaupt verstanden.

Die Spalte ist median, geht durch den ganzen weichen Gaumen und bis zur Mitte des harten Gaumens. Der harte Gaumen ist sehr steil und die beiden Gaumenhälften stehen fast senkrecht. Die Breite der Spalte beträgt in der Mitte $2 \mathrm{~cm}$.

Uranostaphylorshaphie olne Narkose in aufrechter Stellung. 7 Nähte. Vollständige Vereinigung.

Patient wurde sofort mit einem Brugger'schen Obturator versehen und erhielt nun 7 Wochen lang Sprechunterricht bei Herrn Wang. Das Resultat war ein glänzendes: Die Vocale waren nachher ohne allen und jeden nasalen Beiklang Der Spiegel beschlug sich bei a, e, o, u nicht, bei i sehr wenig. Auch die Consonanten hatten ohne Ausnahme das nasale Timbre verloren. Er las nun deutlieh mit klangvoller Stimme und sang fehlerfrei seine Schulbuchlieder. Der Obturator wird ohne Beeinträchtigung der Sprache nach 3 Jahren abgelegt.

8. Sch., Lydia, 9 Jahre alt, aufgenommen den 10. April 1889. Unter den Angehörigen der grossen Verwandtschaft des Kindes findet sich kein Fall von Hasenscharte oder Wolfsrachen. Schon vor 3 Jahren wollten die Eltern das Kind operiren lassen, aber die Aerzte fanden es noch zu jung für eine Operation. 
Intelligentes, graciles Mädchen. Mediane Spaltung des Velum und des harten Gaumens bis zur Mitte. Die Breite der Spalte beträgt im harten Gaumen 13, an der Grenze des harten und weichen Gaumens $19 \mathrm{~mm}$, die Zäpfehenhälften stehen $13 \mathrm{~mm}$ weit von einander. Stark nasale Sprache.

Uranostaphylorrhaphie am 15. April 1889. Anfrischung der Ränder in aufrechter Stellung nach Cocainisirung der Schleimhaut. Dann in Narkose am hängenden Kopf Loslösung der Lappen sammt Naht. Vollständige Vereinigung. Massage und Faradisation des Velum. Dann wurde ein Brugger'scher Obturator angefertigt und mit Hilfe dieses und eines 7wöchentlichen Sprechunterrichts kam Patientin zu einer klangvollen, normalen Sprache. Den Obturator konnte sie später ablegen.

9. 0., Arnold, aus gesunder Familie stammend, in der Missbildungen unbekannt sind, hat von Geburt an eine linksseitige Hasenscharte, die nur bis zur Mitte der Oberippe reicht und eine mediane Gaumenspalte, die, das ganze Velum trennend, bis zur Mitte der Process. palatini reicht. Die Hasenscharte wurde an dem 10 Tage alten Kinde operirt. Zur Operation der Gaumenspalte wurde Patient an 8. April 1889 im Alter von 11 Jahren in's Spital aufgenommen. Die Spalte ist in der Mitte des harten Gaumens $6 \mathrm{~mm}$, an der Grenze des harten und weichen Gaumens $10 \mathrm{~mm}$ und zwischen den Uvulahälften $9 \mathrm{~mm}$ breit. Offenbar bestand frühır eine Medianspalte des ganzen harten Gaumens, denn zwischen den inneren 2 Schneidezähnen beginnt eine in gerader Richtung nach hinten verlaufende, flächenförmige, weisse Narbe, die in der Spitze der Gaumenspalte endigt. Die Sprache ist stark nasal und schwer verständlich. Von den Vocalen e und i stark nasal, $\mathrm{z}$, sch, $\mathrm{t}$ mit schnaubendem Durelischlag durch die Nase, $\mathrm{b}=\mathrm{me}$, $\mathrm{g}=\mathrm{e}$ u. s. w.

Uranostaphylorrhaphie an 17. April 1889. Anfrischung der Ränder in aufrechter Stellung mit Cocain, die ïbrigen Acte der Operation in Chloroformnarkose am hängenden Kopf, 7 Nähte. 1 Stunde p. operat. ziemziemlich starke Nachblutung aus einem Seitensehnitt, die durelı eine halbstündige Digitalcompression endgiltig gestillt wird. Es gelingt die Vereinigung der Spalte bis auf ein bohnengrosses Loch an der Grenze des harten und weichen Gaumens, das durch eine Nachoperation am S. Mai geschlossen wird.

Nach Schluss der Wunde bekommt Patient einen Brugger'schen Obturator und erhält einen 4 wöchentlichen Sprechunterricht. Die Sprache ist nachher normal und der Obturator wird später abgelegt. Dem Sprechunterricht ging eine 14 tägige Our mit Massage und Faradisation des weichen Gaumens voraus.

10. W., Auguste, 13 Jahre alt, aufgenommen den 3. Juni 1889. Die 6 Geschwister der Kranken sind gesund und Missbildungen sind in der Familie unbekannt. Die Spalte wurde in den ersten 9 Lebenstagen dadurch entdeckt, dass das Kind, mit der Flasche genährt, einen Theil der Mileh durch die Nase entleerte. Sie musste mit dem Löffel eingebracht werden. Sie lernte erst mit 2 Jahren sprechen.

Der weiche Gaumen ist in der Mitte gespalten und zwar beträgt die Breite der Spalte zwisehen den Zäpfchenhälften $1,5 \mathrm{~cm}$, in der Mitte desgleichen, weiter oben $1 \mathrm{~cm}$. Die Oberlippe zeigt links vom Infundibulum eine leichte Einkerbung, an der der rothe Lippensaum fehlt. 
Das gutgenährte, blühende und intelligente Mädchen liesst fliessend, aber stark nasal und beinahe unverständlich. Sämmtliche Vocale nasal. Einige Consonanten, wie $\mathrm{k}$ und $\mathrm{v}$ fehlen, $\mathrm{h}, \mathrm{z}$ und ch mit Nasendurehschlag.

Uranostaphylorrhaphie am 3. Juni 1889. 6 Nälıte, olne Narkose mit Cocain in aufrechter Stellung. Am 10. Juni kann die complete Vereinigung constatirt werden. Dann Massage und Faradisation des weichen Gaumens, dann 5 wöchentlicher Sprechunterricht mit Brugger'schem Obturator. Sprache normal, auch nach dem bald erfolgten Ablegen des Obturators.

11. W., Paul, 10 Jahre alt, anfgenommen den 24. Juni 1889. Kind gesunder Eltern, hat eine angeborene Spalte des weichen Gaumens. Die Sprache ist mühsam anzuhören, stark nasal. Das Zungen- $R$ ist nicht vorhanden. Die Buchstaben $\mathrm{s}, \mathrm{f}, \mathrm{z}$ kann es nicht aussprechen. Rümpfen der Nase beim Sprechen.

Uranostaphylorrhaphie am 26. November 1889. Die Operation gelingt auf's Beste. Nach 14 tägigem Sprechunterricht mit Brugger'schem Obturator und Massage des Velum ist die Sprache absolut normal und klangvoll, sämmtliche Vocale sind rein, desgleichen die Consonanten, s, f, z, beide R. Die Bewegungen des Gaumensegels sind kräftig und ausgiebig. Nach weiteren 8 Tagen wird der Obturator entfernt, ohne dass die Sprache im Mindesten Schaden gelitten hätte.

12. Th., Wilhelmine, 12 Jahre alt. Körperlich ordentlich entwickeltes, aber taubstummes Mädehen. Es besteht eine angeborene Spalte des weichen Gaumens mit abgerundetem Winkel gegen den harten Gaumen. Pharynxwulst klein, Hypertrophie der linken Tonsille Die beiden Zäpfehenhälften stehen $22 \mathrm{~mm}$ auseinander. Die Sprache ist unverständlich, monoton und stark näselnd. Sämmtliche Vocale nasal, $\mathrm{k}=\mathrm{h}, \mathrm{g}=\mathrm{e}$. Der Lippenverschluss ist nicht kräftig genug, um einen eigentlichen Explosivlaut zu erzeugen. ch gleich he. Gaumen- $\mathrm{R}$ guttural, Zungen- $\mathrm{R}$ unmöglich. Am 24. October 1890 Uranostaphylorrhaphie am hängenden Kopf in Chloroformnarkose mit Billro th 'scher Modification. 6 Nähte und 2 Nähte an der Rückseite der Uvula. Am 28. ist die Nahtlinie wieder auseinander gegangen mit Ausnahme des Zäpfchens. Am 30. ist auch das Zäpfehen wieder getrennt. Am 1. Januar 1891 nochmalige Operation am hängenden Kopf in Chloroformnarkose. 9 Nähte, 2 am Zäpfehen. Diesmal gelingt die Vereinigung vollständig.

Leider bekam das Mädchen besonderer Verlältnisse halber keinen systematischen Spreehunterricht und so blieb die Sprache nasal, wenn auch verständlicher, als früher. Dieses mässige Resultat ist durch Massage und Faradisation des weichen Gaumens erreicht worden.

Nr. 13 siehe oben.

14. H., Hans, 8 Jahre alt, aufgenommen den 6. August 1891. In der Familie keine Missbildungen. Kam mit linksseitiger Hasenscharte, die im frühen Kindesalter operirt wurde und mit durchgehender linksseitiger Gaumenspalte zur Welt, die an Stelle des 2. fehlenden Schneidezahnes im Process. alveolaris beginnt und das Velum in der Mitte spaltet. Breite der Spalte vorn 5 mun, an der Grenze zwischen hartem und weichem Gaumen $19 \mathrm{~mm}$, in der Mitte des weichen Gaumens $21 \mathrm{~mm}$. Sprache nasal und schwer verständlich. Sämmtliche Vocale nasal, $\mathrm{p}, \mathrm{t}, \mathrm{k}$ haben zischenden Nasendurehschlag. Rein sind nur $1, \ldots 1$ und $n$. 
Uranostaphylorrhaphie am 12. August 1891 in Chloroformnarkose am längenden Kopf. 10 Nähte, 1 an der Hinterseite der Uvula. 1 Stunde post operat. starke Nachblutung aus der Art. palatin. anter. dextr., die durch eine einstündige Digitalcompression endlich zum Stehen gebracht wird. Vollständiger Schluss der Spalte bis auf ein kirschkerngrosses Loch im weichen Gaumen. Am 21. September operativer Schluss dieses Loches und des vordersten Theils der Spalte im Alveolarfortsatz, der der Ernährungsbrüeken wegen nicht bei der ersten Operation vereinigt werden konnte.

Herr Dr. Elmiger schreibt an 13. Juni 1901 über diesen Knaben: Ich massirte den Knaben nach der Operation 3 Monate lang täglich und gab ihm jeden Tag $1 / 2-1$ Stunde Sprechunterricht. Als ich ihn vor 1 Jahre zum letzten Mal sah, sprach er sehr deutlich und ohne nasalen Beiklang. Er sang auch mit klangvoller Stimme. Der selr intelligente Knabe machte seine Schulprüfungen mit Auszeichnung, war der Liebling seiner Lehrer und ist jetzt in einer Bank angestellt.

15. Th.,Ernst, von Meersburg, 12 Jahre alt, aufgenommen den 7. Juli 1895. Gutgenährter, ziemlich kräftiger, taubstummer Knabe, der eine mediane Spalte des weichen Gaumens mit Spaltung der horizontalen Platte des Gaunenbeins auf die Welt brachte.

Am 10. Juli 1895 Uranostaphylorhhaphie am hängenden Kopf in Chloroformnarkose. 7 Nähte und 2 an der Hinterseite der Uvula. Die Vereinigung erfolgt auf's Sehönste und das Resultat ist ein bewegliches Velum mit schön geschwungenen Gaumenbögen. Nach 8 monatlichem Sprechunterricht in der Taubstummenanstalt Meersburg ist die vorher nasale, kaum verständliche Sprache normal geworden ohne Zuhilfenahme eines Obturators.

16. Z., Heinrich, 8 Jahre alt, von Langnau, aufgenommen den 10. April 1896, leidet an einer angeborenen Spalte des weichen Gaumens, war aber im Uebrigen immer gesund. Die Spalte ist in der Mitte $12 \mathrm{~mm}$ breit, am unteren Ende $20 \mathrm{~mm}$. Der Knabe spricht ausserordentlich undeutlich und wurde wegen seiner schlechten Sprache oft und viel von seinen Mitschülern ausgelacht. Sämmtliche Vocale stark nasal, $\mathrm{g}$ und $\mathrm{d}$ existiren nicht, $\mathrm{t}$ und $\mathrm{z}$ haben starken Nasendurchschlag. Flüssige Speisen, wie Milch und Wein, regurgitiren dureh die Nase.

Uranostaphylorrhaphie in Chloroformnarkose am hängenden Kopf den 11. April 1896. Die Vereinigung gelingt bis auf die Zäpfchenspitze.

Auf Anfrage hatte Herr Dr. M eier in Thalweil die Liebenswürdigkeit, mir am 12. Juni 1901 über den Knaben zu berichten, wie folgt: „Z., Heinrich, hat sich weder einen Obturator einsetzen lassen, noeh hat er systematischen Sprechunterricht gehabt. Er hat die Schule mit mittelmässigem Erfolg besucht, der auf seine Flüchtigkeit zurückzuführen ist. Leider hatte er in den ersten Jahren einen schlechten Lehrer (Alkoholiker), der sich nicht die Mühe gab, den Knaben zu richtigem Sprechen zu erziehen, andernfalls wäre das Resultat das denkbar beste, denn wenn er auch jetzt bei raschem Lesen ziemlich näselt, so kann er doch, wenn er sich Mühe giebt und langsam spricht, sehr gut sprechen, so dass man dann nur bei g und $\mathrm{k}$ einen näselnden Ton herausfindet, alles Andere kann er normal sprechen. Spricht er langsam, so merkt ihm ein ungeübtes $\mathrm{Ohr}$ sehr wenig an. Das Resultat ist ein sehr gutes zu nennen."

17. V., Paul, Gymnasiast, 14 Jahre alt, aufgenommen den 5. Juli 1900. 
Intelligenter Knabe, der mit einer durchgehenden, rechtsseitigen Gaumenspalte und rechtsseitiger durchgehender Hasenscharte das Licht der Welt erblickte. Die Hasenscharte wurde im 1. Lebensjahr von mir erfolgreich operirt. Zur Operation der Gaumenspalte konnten sich die Eltern erst jetzt verstehen. In der Familie sind Missbildungen nicht bekannt. Patient ist gross und schlank, von gesundem Aussehen. In der rechten Oberlippe eine alte Narbe, die rechts von der Mittellinie von der Lippe bis zum Nasenloch führt. Der Kiefer total gespalten, die Spalte im Process. alveolaris kaum 1-2 mm breit. Aus dieser Spalte bricht in fast horizontaler Richtung ein Zahn hervor, darüber sitzt ein kleiner, unregelmässiger Wulst, offenbar durch Schrägstellung des Zwischenkiefers im embryonalen Leben entstanden. Die die Spalte einrahmenden Process. palatini sind fast senkrecht gestellt. Die Spalte selbst ist mitten im harten Gaumen $1 \mathrm{~cm}$, an der Grenze des harten und weichen Gaumens $18 \mathrm{~mm}$ breit, die Zäpfchenhälften stehen bei ruhigem Athmen $26 \mathrm{~mm}$ auseinander. Pharynxwulst gut entwickelt. Die Zähne des Unterkiefers ïberragen beim Kieferschluss die Zähne des Oberkiefers, wodurch das Gesicht einen verkniffenen Ausdruck erhält. Die Sprache ist mühsam anzuhören, schwer verständlich, stark näselnd. Sämmtliche Vocale nasal, b, t, s, z mit zischendem Nasendurchschlag, desgleichen h, $\mathrm{g}$ und $\mathrm{k}$ sind nicht vorhanden.

Uranostaphylorrhaphie am 7. Juli 1901 in Chloroformnarkose am hängenden Kopf. 7 Nähte, 2 auf der Hinterseite der Uvulahälften. Die vordere Partie des linken mucösperiostolen Lappen ist unmittelbar nach der Operation bläulichblass, erholt sich aber gegen Abend wieder. In Laufe des Nachmittags eine stärkere Nachblutung aus der linken Art. palatin. anter., die nur durch Fingercompression endgiltig gestillt werden kann. Die Vereinigung gelingt bis auf die vorderste Partie der Spalte im Bereich des Process. alveolaris, die aus schonung für die Ernährungsbrücken überhaupt nicht genäht worden war. Der Defect vorne im harten Gaumen wurde vor der Hand durch eine Kautschukplatte gedeckt, ein Bru g g er'scher Obturator eingesetzt und Patient hatte 4 Wochen Sprechunterricht. Die Sprache war sehr gebessert, aber noch nicht normal. Nach $1 / 2$ Jahr Ablegung des Obturators und nochmaliger kurzer Sprechunterricht. Jetzt spricht und liest Patient mit angenehmer, wohllautender Stimme. August 1902 Schluss des Loches im Bereich des Alveolarfortsatzes.

18. S., Martha, $13 \%$ Jahre alt, aufgenommen den 24. October 1889. Durchgehende linksseitige Gaumenspalte. Die linksseitige Hasenscharte wurde in frithester Jugend (14 Tage) erfolgreich operirt, doch besteht eine gewulstete vorstehende Narbe. Sprache fast unverständlich, nasal. Die Breite der Spalte auf der Höhe der Eckzähne $13 \mathrm{~mm}$, an der Grenze des harten und weichen Gaumens $23 \mathrm{~mm}$. Die Uvulahälften stehen $25 \mathrm{~mm}$ auseinander.

Am 28. October 1889 Uranostaphylorrhaphie. Anfrischung in aufrechter Stellung mit Cocain, die ïbrige Operation in Narkose am hängenden Kopf. Bill r o th 'sche Abmeisselung der Process. pterygoid. 12 Nähte, 2 an der Uvula. Am 16. November Correctur der Hasenscharte und am 2. November 1890 wird noch das kleine Loch dicht hinter den Schneidezähnen zugenält. Dann wurde ein Brugger'scher Obturator angesetzt und 4 Wochen lang Sprechunterricht genommen. Nachher normale Sprache, auch nachdem 
der Obturator bleibend beseitigt worden war. Der Obturator musste in diesem Falle jeweils Nachts weggelegt werden, weil er die Athmung behinderte.

Nr. 19 siehe oben.

20. T., Josephine, 12 Jahre alt, aufgenommen den 3. October 1891. In der Familie keine Bildungsfehler. Durchgehende rechtsseitige Gaumenspalte mit rechtsseitiger Hasenscharte, die im Alter von 12 Tagen operirt wurde. An deren Stelle findet sich jetzt eine lineäre, schiefe, etwas vorragende Narbe. Die Spalte ist an der Grenze des harten und weichen Gaumens $18 \mathrm{~mm}$ breit. Die beiden Zäpfchenhälften stehen $16 \mathrm{~mm}$ auseinander. Die Sprache ist nasal, aber verständlich. Sämmtliche Vocale nasal. $p$, $d$, $f$ und $s$ mit pfeifendem Nasendurchschlag, $k$ und $h$ fehlen $u$. s. w.

Uranostaphylorrhaphie am 3. October 1891 am hängenden Kopf in Chloroformnarkose, 10 Nähte, 2 an der Hinterseite der Uvula. Die Vereinigung gelingt bis auf eine schmale, $2 \mathrm{~mm}$ breite und $1 \mathrm{~cm}$ lange Spalte im harten Gaumen, die sich später von selbst schliesst. Patient erlält nun einen Brugger'schen Obturator und 6 wöehentlichen Sprechunterricht in Meersburg. Nach diesem Unterricht wird die Sprache normal. Später wird der Obturator 2 mal verjüngt und dann abgelegt. Spricht auch ohne Obturator normal.

21. R., Ernst, 12 Jahre alt. Angeborene Spalte des weichen Gaumens und der hinteren Hälfte des harten Gaumens. Sehr nasale, schwer verständliche Sprache.

Uranostaphylorrhaphie am 28. Juni 1892 in Chloroformnarkose am hängenden Kopf, 12 Nähte, 2 an der Hinterseite des Zäpfchens. Heilung gelingt bis auf ein kleines Loch an der Grenze des harten und weichen Gaumens, das durch eine zweite Operation am 26. Juli 1892 gesehlossen wird. Dann Obturator von $\mathrm{Brugger}$, der allmählich verjüngt und dann abgelegt wurde, mit Sprechunterricht 10 Wochen lang. Das Resultat ist eine normale Sprache.

22. H., Ernst, 9 Jahre alt, am 15. Juli 1893 mit einer angeborenen Spalte des weichen Gaumens aufgenommen.

Uranostaphylorrhaphie am 15. Juli 1893, 6 Nähte, 2 an der Hinterseite des Zäpfehens. Vereinigung bis auf die hinterste Partie. Nachoperation am 30. August 1893, womit das ganze Velum hergestellt wird.

Nach einer gütigen Mittheilung des Hausarztes Dr. Isler war die Sprache nach der Operation ohne Sprechunterricht und Obturator bedeutend deutlicher. Der Knabe starb $1890^{\circ}$ an Epityphlitis.

23. H., Johann, 11 Jahire alt, aufgenommen den 25. Mai 1894. In der Familie keine Missbildungen. Patient lernte erst mit 4 Jahren sprechen, besuchte mit 6 Jahren die Seluule und machte gute Fortschritte. Gracil gebauter, etwas schwächlicher Patient. Mediane Spalte des ganzen, weichen Gaumens, deren grösste Breite $17 \mathrm{~mm}$ beträgt. Zusammenhängendes Sprechen und Lesen ist schwer verständlich, und die Sprache ist stark nasal. Sämfntliche Vocale nasal, $g$ existirt nicht, $d, t, z$, auch $f$ mit starkem Nasendurchschlag.

Uranostaphylorrhaphie am 12. Juni 1894 in Chloroformnarkose am hängenden Kopf, 2 Stunden nach der Operation Nachblutung aus einem Seitenschnitt, die durch Compression gestillt wird. 8 Nähte. Nach 4 Tagen bildet sich in Folge localisirter Gangrän ein kleines Loch in der Nahtlinie, sonst gute Vereinigung. Entfernung der letzten Nähte am 7. Tage. Das 
kleine Loch schliesst sich von selbst. Massige des Velum. Nach 6 wöchentlichem Sprechunterriclit in Meersburg olne Obturator ist die Sprache normal.

24. Sch., Conrad, 14 Jahre alt, aufgenommen den 30. Juni 1894. In der Familie keine Sprachstörungen oder Missbildungen. Patient ist stark skoliotisch, sonst gesund und kräftig gebaut. Der weiche Gaumen median gespalten, doch geht die Spalte nicht bis zur Grenze des harten und weichen Gaumens, sondern endet $1 \mathrm{~cm}$ vor derselben. Sprache ziemlich schwer verständlich und nasal Von den Vocalen e und $\mathrm{i}$ stark nasal. $\mathrm{g}$ und $\mathrm{k}$ fallen aus, d, t, s und z rnit Nasendurchschlag.

Uranostaphylorthaphie am 9. Juli 1894 am hängenden Kopf in Narkose, 5 Nante. Die Vereinigung ertolyt aul's Selünste. Nun tägliche Massage des weichen Gaumens während 3 Wochen, dann 4 wöchentlicher Spreclıunterricht in Meersburg, wonach die Sprache normal ist.

25. W., Oscar, 10 Jahre alt, aufgenommen den 17. Juni 1895. Kam mit linksseitiger Hasenscharte und durehgehender Gaumenspalte zur Welt. Die Lippenspalte wurde im 1. Lebensjahre operirt. Gaumenspalte fast durchwegs $2 \frac{1}{2}$ cm breit, die Process. palatini der Oberkiefer sehr schmal. Sprache sehr nasal und schwer verständlich.

Uranostaphylormaphie am 17. Juni 1895 in Narkose am hängenden Kopf, 10 Nälte, 2 an der Rückseite des Zäpfchens. Vollständige Vereinigung. Die Ausbildung der Sprache ist sehr erschwett durch eine bedeutende Herabsetzung des Gehôrs und grosse Sebwächichliceit des Patienten, foch gelingt es mit Hilfe eines Obturators, der melırmals verjüngt und schliesslicls abgelegt wurde und durch 4 monatlichen Sprechunterricht schliesslich eine normale Sprache herzustellen. Patient ist jetzt in der französischen Schweiz in einer Handelsschule und hat auch mit der Aussprache des Französischen keine Schwierjgkeitej.

26. B., Anna, $7^{1 / 2}$ Jahre alt, aufgenommen den 15 . Juli 1895 , hat eine angeborene Spalte des weichen Gaumens von ungewöhnlicher Breite und spricht stark näselnd und schwer verständlich.

Uranostaphylochaphie am 15. Juli 1895 am liängenden Kopf in Chloroformnarkose. Schluss der Spalte bis auf ein erbsengrosses Loch. Ein späteres Bericht lautet, dass die Kranke nie gründlichen Sprechunterricht hatte, auch nie einen Obturator trug. Sie spreche deutlich, aber näsele noch.

27. H., Minna, 11 Jahre alt, aufgenommen den 28. October 1901 , wurde mit einer Spaltung des weichen Gaumens ohne Hasenscharte geboren. Ein Vetter des Kindes hat ebenfalls eine Gaumenspalte. Das Kind ging mit 6 Jahren zur Schule, lernte gut, wurde aber von den Mitschülerinnen wegen der näselnden Sprache oft ausgelacht. Der weiche Gaumen und die horizontale Platte des Gaumenbeins sind gespalten, der harte Gaumen sehr steil. Die Breite der Spalte an der Grenze des harten und weichen Gaumens beträgt $2 \mathrm{~cm}$, zwischen den Uvulahälften bei ruhiger Stellung $2,9 \mathrm{~cm}$. Es besteht nur erre Andentung des Pharyaxwulstes. Sprache stark näselnd, aber noch verständlich.

Uranostaphylorrlaphie am 30. October 1901. Die Vereinigung gelingt bis auf ein bohnengrosses Loch an der Grenze des harten und weichen Gaumens, das später geschlossen wird. Hat noch keinen Sprechunterricht genommen. 